\title{
Color Confinement, Quark Pair Creation and Dynamical Chiral-Symmetry Breaking in the Dual Ginzburg-Landau Theory
}

\author{
H. Suganuma ${ }^{a}$, S. Sasaki ${ }^{\text {b) }}$ and H. Toki ${ }^{\mathrm{a}, \mathrm{b})}$ \\ a) The Institute of Physical and Chemical Research (RIKEN), \\ Hirosawa 2-1, Wako, Saitama 351-01, Japan \\ b) Department of Physics, Tokyo Metropolitan University, \\ Minami-Ohsawa 1-1, Hachioji, Tokyo 192-03, Japan
}

\begin{abstract}
We study the color confinement, the $q-\bar{q}$ pair creation and the dynamical chiralsymmetry breaking of nonperturbative QCD by using the dual Ginzburg-Landau theory, where QCD-monopole condensation plays an essential role on the nonperturbative dynamics in the infrared region. As a result of the dual Meissner effect, the linear static quark potential, which characterizes the quark confinement, is obtained in the long distance within the quenched approximation. We obtain a simple expression for the string tension similar to the energy per unit length of a vortex in the superconductivity physics. The dynamical effect of light quarks on the quark confining potential is investigated in terms of the infrared screening effect due to the $q-\bar{q}$ pair creation or the cut of the hadronic string. The screening length of the potential is estimated by using the Schwinger formula for the $q-\bar{q}$ pair creation. We introduce the corresponding infrared cutoff to the strong long-range correlation factor in the gluon propagator as a dynamical effect of light quarks, and obtain a compact formula of the quark potential including the screening effect in the infrared region. We investigate the dynamical chiral-symmetry breaking by using the Schwinger-Dyson equation, where the gluon propagator includes the nonperturbative effect related to the color confinement. We find a large enhancement of the chiral-symmetry breaking when the dual Meissner effect takes place, which supports the close relation between the color confinement and the chiral-symmetry breaking. The dynamical quark mass, the pion decay constant and the quark condensate are well reproduced by using the consistent values of the gauge coupling constant and the QCD scale parameter with the perturbative QCD and the quark confining potential. The confinement of light quarks is also investigated by the smooth extrapolation of the quark mass function to the time-like momentum region. We find the disappearance of physical poles in the light-quark propagator, and show the confinement of light quarks in the dual Ginzburg-Landau theory.
\end{abstract}




\section{Introduction}

The quantum chromodynamics (QCD) has been accepted as the fundamental theory of strong interactions. Due to the phenomena of the asymptotic freedom in QCD, the ordinary perturbative technique is valid and useful for the analysis of the high-energy hadron reactions with much success [1]. On the contrary, QCD becomes the strong-coupling gauge theory, and exhibits rich phenomena in relation with the nonperturbative nature in the low-energy region. In particular, the color confinement and the dynamical chiral-symmetry breaking are unique features in the nonperturbative region of QCD, and have been studied with much interest by using various strategies, e.g. the effective-model approach or the lattice QCD.

In the perturbative $\mathrm{QCD}$, the QCD scale parameter $\Lambda_{\mathrm{QCD}}$ is one of the most important quantities, and provides a typical scale of the strong interaction. Using the one-loop level perturbation, one obtains the running coupling constant in QCD at a large momentum scale $p^{2}$,

$$
\alpha_{s}\left(p^{2}\right) \equiv \frac{e^{2}\left(p^{2}\right)}{4 \pi}=\frac{12 \pi}{\left(11 N_{c}-2 N_{f}\right) \ln \left(p^{2} / \Lambda_{\mathrm{QCD}}^{2}\right)},
$$

where $N_{c}$ and $N_{f}$ are the numbers of the color and flavor, respectively [1]. In the infrared region, this running coupling constant becomes large and formally diverges at $p^{2}=\Lambda_{\mathrm{QCD}}^{2}$, so that the simple perturbation theory is no more meaningful and some nonperturbative effect should appear instead. Some experimental data suggests $\alpha_{s}\left(m_{Z}^{2}\right) \simeq 0.108 \pm 0.005$ from the analysis of the recent LEP data [2], or $\alpha_{s}\left((35 \mathrm{GeV})^{2}\right)=0.10 \sim 0.17$ from the high-energy lepton-hadron reaction [1]. The QCD scale parameter $\Lambda_{\mathrm{QCD}}$ is obtained from these experimental data, and its recent value is given as $\Lambda_{\mathrm{QCD}}(\overline{\mathrm{MS}})=220 \pm 15 \pm 50 \mathrm{MeV}$ in the $\overline{\mathrm{MS}}$ scheme [1] from the experimental data of the BCDMS collaboration [3]. Hence, one roughly estimates from Eq. $(1.1), \alpha_{s}\left((0.5 \mathrm{GeV})^{2}\right) \sim 1$, which means the breakdown of the validity of the perturbation theory. Thus, we expect the appearance of the nonperturbative effect in the infrared region, $p \lesssim 1 \mathrm{GeV}$, due to the strong coupling.

Now, we shall give an overview of the dynamical chiral-symmetry breaking and the color confinement as the outstanding nonperturbative features in the infrared region of QCD. Although QCD Lagrangian has the chiral symmetry in the massless quark limit, it is spontaneously broken in the nonperturbative QCD vacuum [4]. In the phenomenological point of view, the dynamical chiral-symmetry breaking leads the absence of the parity doubling for hadrons and the success of the low-energy theorem or the current algebra [5]. The dynamical chiral-symmetry breaking is characterized by the quark condensate, $\langle\bar{q} q\rangle \simeq-(225 \pm 25 \mathrm{MeV})^{3}[6,7]$. Quark condensation is caused by the attractive interaction between quarks, and 
this phenomenon is analogous with Cooper-pair condensation in the superconductivity. Such a physical process has been demonstrated by using the effective models of QCD, for instance, the Nambu-Jona-Lasinio model [8] or the instanton liquid model [7]. The SU(6) theory [1] or the effective-model approaches [7] suggest that light quarks get a large effective mass, $M \simeq 350 \mathrm{MeV}$, and behave as massive constituent quarks in the infrared energy region, as the result of the dynamical chiral-symmetry breaking. The pion is identified as the Nambu-Goldstone boson related to the dynamical chiral-symmetry breaking, and obeys the low-energy theorem or the current algebra [5], where the pion decay constant, $f_{\pi} \simeq 93 \mathrm{MeV}$, is also a relevant quantity characterizing the breaking of chiral symmetry [4]. Thus, the dynamical chiral-symmetry breaking is characterized by several quantities, the chiral condensate $\langle\bar{q} q\rangle \simeq-(225 \pm 25 \mathrm{MeV})^{3}$, the effective quark mass $M \simeq 350 \mathrm{MeV}$, and the pion decay constant $f_{\pi} \simeq 93 \mathrm{MeV}$.

The color confinement is signaled as the absence of the asymptotic states of the colored particles. In the phenomenological point of view, hadrons except the pion are considered to be string-like because of the Regge trajectories of hadrons [9] or the duality of the hadron reaction. The string picture of hadrons suggests a linear potential between quarks, due to which quarks are confined. In particular, the universality of the Regge slopes of hadrons means the universal value of the string tension [9], $k \simeq 1 \mathrm{GeV} / \mathrm{fm}$, which characterizes the strength of the confinement. Then, the question on the confinement is how and why the color-electric flux between quarks is squeezed like a string or a tube. The natural explanation is the exclusion of the color-electric field in the nonperturbative QCD vacuum, where the color dielectric constant vanishes.

In terms of the vanishing of the color dielectric constant, Y. Nambu, 't Hooft and Mandelstam paid attention to an analogy between the color confinement and the Meissner effect, the vanishing of the magnetic permeability and the exclusion of the magnetic flux in the superconductor, and regarded the color confinement as the dual version of the superconductivity [10-12]. Their idea is based on the duality, a kind of the symmetric property between the electric part and the magnetic part in the gauge theories like QED or QCD. In this picture, the dual Meissner effect, the exclusion of the (color-)electric field, is brought by (color-)monopole condensation instead of Cooper-pair condensation in the superconductivity.

The appearance of magnetic monopoles in QCD was investigated by 't Hooft based on the abelian gauge fixing [13], which was assumed to be the relevant gauge fixing to understand the color confinement. The abelian gauge fixing is defined by the diagonalization of an arbitrary gauge-dependent physical variable, and nonabelian gauge theories are generally reduced into abelian gauge theories with magnetic monopoles in this gauge. When such QCD-monopoles are condensed due 
to some attractive force between them, the dual Meissner effect takes place, that is, the color-electric field is excluded in the QCD vacuum. In this case, the colorelectric flux between static quarks are squeezed into an one-dimensional string or a tube like a vortex in the superconductivity. Such a string picture of hadrons leads to a linear potential between static quarks, characterizing the quark confinement, and is consistent with the Regge trajectories of hadrons and the lattice QCD data of the quenched approximation. Thus, QCD-monopole condensation seems to give a physical explanation of the color confinement and related phenomena in the nonperturbative region of QCD.

The Kanazawa group recently formulated the dual Ginzburg-Landau theory as a phenomenological theory for the nonperturbative QCD by introducing the QCD-monopole field and its interaction $[14,15]$ based on 't Hooft's conjecture of QCD-monopole condensation. Within the quenched approximation, they derived the linear quark potential in the long distance, and demonstrated the realization of the dual Meissner effect, e.g., formation of the hadron flux tube between static quarks [16].

In recent years, several authors have pointed out the abelian dominance, i.e. the importance of the abelian degrees of freedom for the nonperturbative quantities in the nonabelian gauge theory based on the lattice gauge simulation [17, 18]. In their studies, the abelian configurations seem to play a dominant role in the nonperturbative quantities like the Wilson loop or the Polyakov loop $[17,18]$, and such an evidence of the abelian dominance seems to lead the relevance of the abelian gauge to the color confinement.

The important role of the QCD-monopole to the confinement have been also studied by using the lattice gauge simulations [18]. In the compact QED, there is the confining phase in the strong-coupling region [19] as well as QCD, and monopole condensation is known to play an essential role to the confinement from the studies of the lattice gauge theory $[20,21]$. In the nonabelian gauge theories, several authors have pointed out the importance of the QCD-monopole to the confinement. Kronfeld et al. [22] compared the SU(2) gauge theory and the compact QED by using the lattice gauge simulation, and showed that monopole condensation seems to take place also in the $\mathrm{SU}(2)$ gauge theory. In Ref.[18], an enhancement of the time component of the monopole current in the deconfinement phase was reported as an evidence of QCD-monopole condensation, which suggests the interesting correspondence between the spatial correlation of the monopoles and the color confinement. Very recently, the Kanazawa group showed that the entropy of an extended monopole loop dominates over its energy and monopole condensation occurs on the renormalized lattice in the infrared region, $\beta<\beta_{c}$, by using the $\mathrm{SU}(2)$ lattice gauge simulation [23]. They also showed that the string tension of 
the $\mathrm{SU}(2)$ gauge theory are well reproduced by extended monopole contributions alone [23]. Thus, several evidences on the abelian dominance and the relevant role of the QCD-monopole to the confinement have been found in the lattice gauge simulation. Of course, much more progress should be made to understand the relation between QCD-monopole condensation and the color confinement.

The dynamical effect of light quarks is also important for the color confinement, because the linear quark potential is screened due to the $q-\bar{q}$ pair creation in the long distance, which means the cut of hadronic strings. The static quark potential seems to be saturated in the infrared region, where the system is described as two mesons including light and heavy fermions. Such a tendency has been observed in the lattice QCD simulation with dynamical quarks [24]. The screening effect on the long-range correlations between quarks corresponds to the reduction of the strong correlation in the infrared momentum region in the gluon propagator. Hence, the gluon propagator should be modified by the $q-\bar{q}$ pair creation effect.

The dynamical chiral-symmetry breaking is also one of the most important properties of the light-quark dynamics, and has been studied by using the SchwingerDyson equation [25-28]. However, most authors used a free gluon propagator in the Schwinger-Dyson equation, and neglected the nonperturbative effect related to the color confinement. In the dual Ginzburg-Landau theory, one can incorporate the nonperturbative effect related to the color confinement in the infrared region to the Schwinger-Dyson equation by using the gluon propagator including the effects of QCD-monopole condensation.

In the dual Ginzburg-Landau theory, the energy region is naturally divided into two kinds of regions corresponding to the perturbative QCD and the nonperturbative QCD. In the ultraviolet region, the perturbative features are expected and the system is described by the use of the running coupling constant at the one or two loop level in the perturbative scheme. On the other hand, the system should be nonperturbative in the infrared region and such a nonperturbative effect is included in the dual Ginzburg-Landau theory as the appearance of QCD-monopole condensation or the dual Meissner effect. Hence, one can study the nonperturbative effect related to the color confinement on the dynamical chiral-symmetry breaking in the dual Ginzburg-Landau theory.

The light-quark confinement can be also investigated by using the SchwingerDyson equation, although this issue is rather difficult because the discussion of the static quark potential cannot be applied to the light quarks unlike the heavy quarks. Instead, the light-quark confinement is characterized by the disappearance of the physical poles in the quark propagator. By using the Schwinger-Dyson equation, one gets the dynamical quark mass $M\left(p^{2}\right)$ depending on the momentum in the space-like region. One can examine the disappearance of the physical poles in the 
propagator by the analytic continuation of $M\left(p^{2}\right)$ from the space-like region to the time-like region.

In this paper, we study the nonperturbative features of QCD, the color confinement, the $q-\bar{q}$ pair creation and the dynamical chiral-symmetry breaking by using the dual Ginzburg-Landau theory as a phenomenological theory based on QCD, which includes the mechanism of QCD-monopole condensation and the dual Meissner effect. We study their mutual relations and construct the unified picture of these important features of the nonperturbative QCD in the infrared region and the perturbative QCD in the ultraviolet region in terms of the dual Ginzburg-Landau theory.

In chapter 2, we review the relation between the abelian gauge fixing and the appearance of the QCD-monopole in line with 't Hooft's study [13]. By the abelian gauge fixing, the nonabelian gauge group is reduced to the abelian one, and abelian monopoles appear as singularities of the residual abelian gauge field.

In chapter 3, we construct the dual Ginzburg-landau theory in line with the Kanazawa group [14,15] by introducing the possible self-interaction between abelian monopoles similar to the Ginzburg-Landau theory in the superconductivity.

In chapter 4, we derive the static quark potential by investigating the correlation between the quark currents within the quenched approximation [14,15]. The quark potential has the linear part and the Yukawa part, and is compared with a phenomenological potential. We obtain a simple expression for the string tension, which is analogous to that of the energy per unit length of the vortex in the superconductivity.

In chapter 5, we study the dynamical effects of the light quarks on the quark confining potential. The linear potential is screened in the long-range region due to the $q-\bar{q}$ pair creation [24]. The screening length of the linear potential is estimated by using the Schwinger formula of the $q-\bar{q}$ pair creation in the hadron flux tube $[29,30]$. By introducing the infrared cutoff in the gluon propagator, the screening effect for the quark potential is reproduced in the long-range region. We obtain a compact formula for the screened quark potential in the dual Ginzburg-Landau theory.

In chapter 6 , we investigate the dynamical chiral-symmetry breaking by the use of the Schwinger-Dyson equation, where the gluon propagator includes both the nonperturbative effect in relation with the confinement and the infrared cutoff due to the $q-\bar{q}$ pair creation effect. Since the double pole of the gluon propagator vanishes due to the infrared cutoff, we can calculate the Schwinger-Dyson equation without any difficulties of the infrared divergence. By solving the Schwinger-Dyson equation within the rainbow approximation, we find a large enhancement of the 
dynamical chiral-symmetry breaking due to QCD-monopole condensation. The physical quantities related to the chiral-symmetry breaking are reproduced by the use of the consistent values for the gauge coupling constant and the QCD scale parameter with the color confinement and the perturbative QCD. By extrapolating the quark mass function $M\left(p^{2}\right)$, which is obtained by the Schwinger-Dyson equation, to the time-like region, we also examine the existence of the pole in the light-quark propagator. We find the disappearance of poles in the light-quark propagator, which means the confinement of the light quark.

Section 7 is devoted to the summary and discussions.

\section{Abelian Gauge Fixing and QCD-Monopoles}

In this chapter, we review the abelian gauge fixing in the nonabelian gauge theories and the appearance of the degree of freedom of the QCD-monopole in line with 't Hooft's work [13].

The color confinement is characterized by the vanishing of the color dielectric constant and the exclusion of the color-electric field in the nonperturbative QCD vacuum. Such features can be explained by the dual Meissner effect caused by magnetic monopole condensation [11, 12], which is the dual version of Cooper-pair condensation in the superconductivity. In this respect, the abelian gauge fixing [13] is one of the most interesting gauge for the study of color confinement, because the degrees of freedom of the magnetic monopole naturally appears in this gauge.

The abelian gauge fixing is defined by the diagonalization of an arbitrary gaugedependent variable, $X(x)=T^{a} X^{a}(x)$, where $T^{a}\left(a=1,2, . ., N_{c}^{2}-1\right)$ denotes the generator of the nonabelian gauge group, $\mathrm{SU}\left(N_{c}\right)$. In this gauge, $X(x)$ is diagonalized by the gauge transformation, in the fundamental representation of $\mathrm{SU}\left(N_{c}\right)$,

$$
X(x) \rightarrow X^{\prime}(x)=\Omega(x) X(x) \Omega^{-1}(x)=\operatorname{diag}\left(\lambda_{1}(x), \lambda_{2}(x), . ., \lambda_{N_{c}}(x)\right) \equiv X_{d}(x),
$$

where $\Omega(x)=\exp \left(i T^{a} \chi^{a}(x)\right) \in \mathrm{SU}\left(N_{c}\right)$ is a gauge function, Minkowski space.

In the abelian gauge, there remains the abelian gauge symmetry of $[\mathrm{U}(1)]^{N_{c}-1}$, the maximal torus subgroup of $\mathrm{SU}\left(N_{c}\right)$, because $X_{d}(x)$ is invariant under the gauge transformation defined by a gauge function, $\omega(x) \in[\mathrm{U}(1)]^{N_{c}-1} \subset \mathrm{SU}\left(N_{c}\right)$, or $\omega(x)=\operatorname{diag}\left(e^{i \psi_{1}(x)}, e^{i \psi_{2}(x)}, . ., e^{i \psi_{N_{c}}(x)}\right)$ with a constraint $\sum_{a=1}^{N_{c}} \psi_{a}=0$, that is, one finds $\omega(x) X_{d}(x) \omega^{-1}(x)=X_{d}(x)$. The off-diagonal elements of the gauge degrees of freedom are frozen, but the diagonal elements of the gluon field remain as the gauge degrees of freedom in the abelian gauge. In other words, nonabelian gauge theories are reduced to the abelian gauge theories by imposing the abelian gauge 
fixing condition, and the off-diagonal gluons are regarded as charged matter fields in terms of the residual abelian gauge symmetry.

Next we show the appearance of QCD-monopoles [13], magnetic monopoles in terms of the residual abelian gauge field, in the abelian gauge. At degeneracy points of the eigenvalues of $X(x)$, the abelian gauge fixing is not unique even for the off-diagonal part, and they behave as singular points of the residual abelian gauge field. Then, these degeneracy points become monopoles with respect to the abelian gauge field. Let us examine the above argument by taking the $N_{c}=2$ case for simplicity, although the generalization to an arbitrary $N_{c}$ case is straightforward.

We investigate the topological nature near the degeneracy points of the eigenvalues of $X(x)=\frac{\tau^{a}}{2} X^{a}(x)$. Since two eigenvalues of $X(x)$ are given by $\pm \frac{1}{2}\left(X_{1}^{2}(x)+\right.$ $\left.X_{2}^{2}(x)+X_{3}^{2}(x)\right)^{1 / 2}$, one finds $X_{1}(x)=X_{2}(x)=X_{3}(x)=0$ at the degeneracy points. Since these three conditions are independent generally, the degeneracy points form the point-like manifolds in the three-dimensional space $\mathbf{R}^{3}$ at each time $t^{\star}$. Let us consider the neighborhood of a degeneracy point $\mathbf{x}_{0} \in \mathbf{R}^{3}$, which satisfies $X\left(\mathbf{x}_{0}\right)=0$. By using the Taylor expansion near $\mathbf{x}_{0}$, one obtains

$$
X(\mathbf{x})=\frac{\tau^{a}}{2} C^{a i}\left(\mathbf{x}-\mathbf{x}_{0}\right)^{i}, \quad C^{a i} \equiv \partial^{i} X^{a}\left(\mathbf{x}_{0}\right),
$$

where $O\left(\left(\mathbf{x}-\mathbf{x}_{0}\right)^{2}\right)$ is neglected. One finds $\operatorname{det} C \neq 0$ when the manifold of the degeneracy points is point-like in $\mathbf{R}^{3}$, which corresponds to the general case.

In order to examine the topology of the gauge field near $\mathbf{x}_{0}$, it is useful to introduce the new space-coordinate variable $\mathbf{w}$ such that $w^{a} \equiv C^{a i}\left(\mathbf{x}-\mathbf{x}_{0}\right)^{i}$ instead of $\mathbf{x}$. From the fact of $\operatorname{det} C \neq 0$ in the general case, such a transformation of the coordinate variable is simply reduced to the linear transformation near the degeneracy point, so that the topological nature is not changed. In terms of the new coordinate $w^{a}$, one finds the hedgehog configuration,

$$
X\left(w^{a}\right)=\frac{\tau^{a}}{2} w^{a}
$$

near the degeneracy point, $w^{a}=0$, and the eigenvalues of $X$ is found to be $\pm \frac{1}{2}\left(w_{1}^{2}+\right.$ $\left.w_{2}^{2}+w_{3}^{2}\right)^{1 / 2}$. This hedgehog configuration (2.3) is one of the simplest nontrivial solution corresponding to the homotopy group $\pi_{2}(\mathrm{SU}(2) / \mathrm{U}(1))=\pi_{1}(\mathrm{U}(1))=Z_{\infty}$, and such a configuration widely appears in the particle physics, for instance, the 't Hooft-Polyakov monopole [31], the instanton in the nonabelian gauge theory [32], and the chiral soliton in the Skyrme-Witten model [33]. It should be noted that such a topological configuration is originated from the nonabelian nature.

* We will consider the system at each $t$, and omit the time variable $t$ hereafter in this chapter. 
By the use of the parametrization such as $\mathbf{w}=\left(w_{1}, w_{2}, w_{3}\right)=(r \cos \phi \sin \theta, r \sin \phi \sin \theta, r \cos \theta), X$ can be expressed as

$$
X=\frac{1}{2}\left(\begin{array}{cc}
w_{3} & w_{1}-i w_{2} \\
w_{1}+i w_{2} & -w_{3}
\end{array}\right)=\frac{r}{2}\left(\begin{array}{cc}
\cos \theta & e^{-i \phi} \sin \theta \\
e^{i \phi} \sin \theta & -\cos \theta
\end{array}\right) .
$$

Hence, the gauge function $\Omega \in \mathrm{SU}(2)$ which diagonalizes $X$ is found to be

$$
\Omega(\mathbf{w})=\left(\begin{array}{cc}
e^{i \phi} \cos \frac{\theta}{2} & \sin \frac{\theta}{2} \\
-\sin \frac{\theta}{2} & e^{-i \phi} \cos \frac{\theta}{2}
\end{array}\right),
$$

which leads $\Omega X \Omega^{-1}=X_{d}$. By the gauge transformation $\Omega$, the gauge field is transformed as

$$
A_{\mu} \rightarrow A_{\mu}^{\prime}=\Omega\left(A_{\mu}-\frac{i}{e} \partial_{\mu}\right) \Omega^{-1},
$$

where $\partial_{\mu} \equiv \frac{\partial}{\partial x^{\mu}}$. Since the original gauge field $A_{\mu}$ is regular, $\Omega A_{\mu} \Omega^{-1}$ is also regular. On the other hand, $A_{\mu}^{s} \equiv-\frac{i}{e} \Omega \partial_{\mu} \Omega^{-1}$ becomes singular, as is shown in the following. Consider the line-integral of $A_{\mu}^{s}$ along a contour $C$,

$$
\begin{aligned}
\Phi & \equiv-\int_{C} A_{\mu}^{s} d x^{\mu}=\int_{C} A_{i}^{s} d x_{i}=-\frac{i}{e} \int_{C} \Omega \partial_{i} \Omega^{-1} d x_{i} \\
& =-\frac{i}{e} \int_{C} \Omega \frac{\partial}{\partial w_{a}} \Omega^{-1} d w_{a}=\int_{C} \mathcal{A}_{a}^{s}(\mathbf{w}) d w_{a},
\end{aligned}
$$

where $\mathcal{A}_{a} \equiv-\frac{i}{e} \Omega(\mathbf{w}) \frac{\partial}{\partial w_{a}} \Omega^{-1}(\mathbf{w})$. When $C$ is a closed loop, $\Phi$ is identified as the magnetic flux which penetrates the area inside the closed contour $C$ by way of the Stokes theorem.

Let $C$ be taken as a closed circle such that $r$ and $\theta$ are constant, while $\phi \in[0,2 \pi)$, for simplicity. Then, one gets

$$
\begin{aligned}
\Phi(\theta) & =-\frac{i}{e} \int_{0}^{2 \pi} d \phi \Omega \frac{\partial}{\partial \phi} \Omega^{-1} \\
& =-\frac{1}{e} \int_{0}^{2 \pi} d \phi\left(\begin{array}{cc}
\cos ^{2} \frac{\theta}{2} & -e^{i \phi} \sin \frac{\theta}{2} \cos \frac{\theta}{2} \\
-e^{-i \phi} \sin \frac{\theta}{2} \cos \frac{\theta}{2} & -\cos ^{2} \frac{\theta}{2}
\end{array}\right) \\
& =-\frac{2 \pi}{e}\left(\begin{array}{cc}
\cos ^{2} \frac{\theta}{2} & 0 \\
0 & -\cos ^{2} \frac{\theta}{2}
\end{array}\right)=-\frac{2 \pi}{e} \cos ^{2} \frac{\theta}{2} \cdot \tau_{3}=-\frac{2 \pi}{e}(1+\cos \theta) \frac{\tau_{3}}{2} .
\end{aligned}
$$

Since the final formula of $\Phi$ is diagonal, $\Phi$ is identified as the magnetic flux of the 
abelian gauge field.

It is notable that $\Phi$ is finite even at $\theta=0$, where contour $C$ shrinks into a point. This means that the corresponding magnetic field diverges at $\theta=0$ like a $\delta$-function, and the gauge field is singular at $\theta=0$. Then, the magnetic flux is decomposed into two parts,

$$
\Phi(\theta)=-\frac{2 \pi}{e}(1+\cos \theta) \frac{\tau_{3}}{2}=\frac{4 \pi}{e}\left\{\frac{1-\cos \theta}{2}-1\right\} \frac{\tau_{3}}{2}
$$

where the first and the second terms in the curly bracket are nothing but the expressions of the magnetic flux of the magnetic monopole with the magnetic charge $g=\frac{4 \pi}{e}$ and the corresponding Dirac string, respectively. The relation $e g=4 \pi$ corresponds to the Dirac condition for magnetic monopoles [32]. Hence, the abelian gauge field near the degeneracy point of eigenvalues of $X$ is equivalent to the magnetic monopole system in the $w$-coordinate space.

Since the topological natures are not changed by the transformation between $x$ and $w$, the degeneracy points also become the magnetic monopoles in the abelian gauge field in terms of the $x$-coordinate space. Thus, the QCD-monopoles appear at the degeneracy point of the eigenvalues of $X$ in the abelian gauge. In this scheme, the degeneracy condition $\operatorname{det} X=0$ is independent of the representation of the nonabelian group. Hence, the number and the location of the abelian magnetic monopole do not depend on the representation for each $X$, although this gauge fixing depends on the selection of the gauge dependent variable $X$.

Thus, nonabelian gauge theories are reduced to abelian gauge theories with QCD-monopoles in the abelian gauge, and the degrees of freedom of QCD-monopoles naturally appear in QCD in this gauge. If the QCD-monopole is condensed like the Cooper pair in the superconductivity theory, one expects the dual Meissner effect [11-13], the vanishing of the color dielectric constant and the exclusion of the color-electric flux, which characterizes the color confinement.

In recent years, several authors found evidences of the abelian dominance, that is the relevant roles of the abelian degrees of freedom to the nonperturbative quantities in the nonabelian gauge theory by using the lattice gauge simulation $[17,18]$. They pointed out that the abelian gauge configurations seems to play a dominant role to the nonperturbative quantities like the Wilson loop or the Polyakov loop in the lattice simulations of the nonabelian gauge theory $[17,18]$. The abelian dominance gives a consistent picture with 't Hooft's conjecture, the relevance of the abelian gauge fixing to the confinement.

The role of the QCD-monopole to the confinement has been also examined by the use of the lattice gauge simulations. The compact QED [19] has a confining phase in the strong-coupling region as well as QCD, and the confinement is known 
to be realized due to monopole condensation [20, 21]. The importance of the QCD-monopole to the confinement has been also reported in the nonabelian gauge theories. Some evidences of QCD-monopole condensation was reported by Kronfeld et al. [22] by comparing the $\mathrm{SU}(2)$ gauge theory and the compact QED in the lattice gauge theories. Another group has reported a drastic change of the space-time asymmetry of the monopole currents at the deconfinement phase transition, which may provide an interesting correspondence between the spatial correlation of the monopoles and the color confinement [18]. Although there is no difference between the time component and the space one of the current in the confining phase, there is a clear enhancement of the time component of the monopole current in the deconfinement phase [18]. This change suggests that the QCD-monopoles become more static in the deconfinement phase, which can be regarded as a reduction of the spatial correlation of the monopoles. Very recently, the Kanazawa group investigated the energy and the entropy of a monopole loop by using the SU(2) lattice gauge simulation [23]. They found that the entropy of an extended monopole loop dominates over its energy and monopole condensation takes place on the renormalized lattice in the infrared region, $\beta<\beta_{c}$ [23]. They also showed that the string tension of the $\mathrm{SU}(2)$ gauge theory are well reproduced by such a extended monopole contributions alone [23]. Thus, recent lattice gauge simulations have given the phenomenological evidences of the abelian dominance and the important role of the QCD-monopole to the color confinement. Hence, 't Hooft's conjecture of QCD-monopole condensation has been accepted as a realistic interpretation of the color confinement in the infrared region.

Finally, we reconsider the role of the nonabelian nature to the appearance of QCD-monopoles in this scheme. It is notable that the off-diagonal part of the gauge field contributes to the QCD-monopole or the hedgehog configuration (2.3) as well as the diagonal part, and the corresponding gauge configuration $A_{\mu}^{\prime}(x)$ in Eq.(2.6) has both the diagonal and the off-diagonal parts, although the colormagnetic flux $\Phi$ in Eq.(2.8) is diagonal. In this respect, these monopoles are regarded as the collective modes including the effect of the off-diagonal gauge field. In the topological point of view, the nonabelian nature of the nonabelian gauge theory plays an essential role on the appearance of the hedgehog configuration (2.3) in the full gauge space corresponding to the nontrivial homotopy group $\pi_{2}\left(\mathrm{SU}\left(N_{c}\right) /[\mathrm{U}(1)]^{N_{c}-1}\right)=\pi_{1}\left([\mathrm{U}(1)]^{N_{c}-1}\right)=Z_{\infty}^{N_{c}-1}[32]$, and this configuration behaves as the singularity or the Dirac monopole in terms of the residual abelian gauge field in the abelian gauge.

It is interesting to compare the superconductivity with the nonabelian gauge system in the abelian gauge. In an ordinary sense, the pure-gauge system seems quite different from the complicated matter system in the superconductor including 
the electron and the metallic lattice. However, even the pure-gauge system of the nonabelian gauge theory may give a similar situation to the superconductor by using the abelian gauge fixing. In the abelian gauge, the diagonal part and the offdiagonal part of the gauge field come to play a different role in a physical meaning. The diagonal gauge fields remain to be gauge fields, while the off-diagonal gauge fields behave as charged matter fields and permit the appearance of the QCDmonopoles as mentioned above. Then, this off-diagonal gauge fields in the abelian gauge may play a similar role to the matter system in the superconductor, and therefore one may expect the similar situation to the complicated matter system of the superconductor.

In the next chapter, we construct the phenomenological theory based on QCD including the dual Meissner effect, and study the nonperturbative phenomena of QCD, not only the confinement but also the $q-\bar{q}$ pair creation and the dynamical chiral-symmetry breaking, based on 't Hooft's conjecture, the relevance of the abelian gauge and the abelian monopoles in the infrared region.

\section{The Dual Ginzburg-Landau Theory and QCD-Monopole Condensation}

In this chapter, we construct the effective theory of the nonperturbative QCD in the abelian gauge, by introducing the QCD-monopole field as a relevant mode responsible to the color confinement. The QCD Lagrangian is described by quarks $q^{\alpha}$ and gluons $A_{\mu}[1]$,

$$
\mathcal{L}_{\mathrm{QCD}}=-\frac{1}{4} G_{\mu \nu}^{a} G_{a}^{\mu \nu}+\bar{q}(i \not D-m) q,
$$

where $G_{\mu \nu} \equiv \partial_{\mu} A_{\nu}-\partial_{\nu} A_{\mu}+i e\left[A_{\mu}, A_{\nu}\right]$ and $D_{\mu} \equiv \partial_{\mu}+i e A_{\mu}$ are the field strength tensor and the covariant derivative, respectively.

We shall take the abelian gauge fixing presented by 't Hooft. There remains the gauge symmetry in the diagonal part of the gauge degrees of freedom in the abelian gauge, so that the diagonal gluons would bring a large contribution to the dynamical process as the gauge fields even in this gauge. On the contrary, offdiagonal gluons behave as charged matter fields instead of the gauge fields because the off-diagonal parts of the gauge degrees of freedom are fixed in this gauge [13]. The appearance of QCD-monopoles is important and should be taken into account in the abelian gauge. We consider the main role of the off-diagonal gluon is to form the QCD-monopoles in this gauge [14], which was supported by the recent Monte Carlo simulation $[17,18,23]$. The infrared effective theory of QCD is thus constructed by the abelian gauge fields and the QCD-monopole fields in the abelian gauge. 
To begin with, let us consider the abelian gauge part in the effective theory,

$$
\mathcal{L}_{\text {Abel }} \equiv-\frac{1}{4} \vec{f}_{\mu \nu}^{2}+\bar{q}(i \not \partial-e \vec{A} \cdot \vec{H}-m) q
$$

where $\vec{f}_{\mu \nu} \equiv \partial_{\mu} \vec{A}_{\nu}-\partial_{\nu} \vec{A}_{\mu}, \vec{H} \equiv\left(T_{3}, T_{8}\right)$, and $\vec{A}^{\mu} \equiv\left(A_{3}^{\mu}, A_{8}^{\mu}\right)$. Owing to the duality of the abelian gauge theory, one can introduce the QCD-monopole field and study the dual Meissner effect by the parallel argument to the superconductivity. To make the duality of the gauge theory manifest, it is convenient to use the Zwanziger form [34],

$$
\begin{aligned}
-\frac{1}{4} \vec{f}_{\mu \nu}^{2} & =-\frac{1}{4}\left(\partial_{\mu} \vec{A}_{\nu}-\partial_{\nu} \vec{A}_{\mu}\right)^{2}=-\frac{1}{4}\left(\partial_{\mu} \vec{B}_{\nu}-\partial_{\nu} \vec{B}_{\mu}\right)^{2} \\
& =-\frac{1}{2 n^{2}}[n \cdot(\partial \wedge \vec{A})]^{\nu}[n \cdot *(\partial \wedge \vec{B})]_{\nu}+\frac{1}{2 n^{2}}[n \cdot(\partial \wedge \vec{B})]^{\nu}\left[n \cdot *^{*}(\partial \wedge \vec{A})\right]_{\nu} \\
& -\frac{1}{2 n^{2}}[n \cdot(\partial \wedge \vec{A})]^{2}-\frac{1}{2 n^{2}}[n \cdot(\partial \wedge \vec{B})]^{2},
\end{aligned}
$$

where $n_{\mu}$ is an arbitrary constant four vector, corresponding to the direction of the Dirac string, $[a \cdot(b \wedge c)]^{\nu} \equiv a_{\mu}\left(b^{\mu} c^{\nu}-b^{\nu} c^{\mu}\right)$, and $\left[a^{*}(b \wedge c)\right]^{\nu} \equiv a_{\mu} \epsilon^{\mu \nu \alpha \beta}\left(b_{\alpha} c_{\beta}\right)$. The field denoted by $\vec{B}_{\mu}$ is called the dual gauge field, because it satisfies the relation

$$
{ }^{*} \vec{f}_{\mu \nu}=\partial_{\mu} \vec{B}_{\nu}-\partial_{\nu} \vec{B}_{\mu}
$$

in the absence of the electric current. Then, the Lorentz indices of the electric and magnetic fields are interchanged each other by using the dual gauge field $\vec{B}_{\mu}$, instead of $\vec{A}_{\mu}$ in this case.

In the Zwanziger form, the magnetic current $\vec{k}_{\mu}$ directly couples with the dual gauge field $\vec{B}_{\mu}$ [34], similar to the coupling between the electric current $\vec{j}_{\mu}$ and the ordinary gauge field $\vec{A}_{\mu}$. Hence, the interaction term in the presence of the electric current and the magnetic current is given by $\mathcal{L}_{\text {int }}=\vec{j}_{\mu} \vec{A}^{\mu}+\vec{k}_{\mu} \vec{B}^{\mu}$.

It is notable that the last expression in Eq.(3.3) is invariant under the two types of the local transformations,

$$
\begin{aligned}
\vec{A}_{\mu}(x) & \rightarrow \vec{A}_{\mu}(x)+\frac{1}{e} \partial_{\mu} \vec{\theta}_{A}(x), \\
\vec{B}_{\mu}(x) & \rightarrow \vec{B}_{\mu}(x)+\frac{1}{g} \partial_{\mu} \vec{\theta}_{B}(x),
\end{aligned}
$$

where $\theta_{A}(x)$ and $\theta_{B}(x)$ are independent arbitrary scalar functions. Then, we see the extended local symmetry, $\left[\mathrm{U}(1)_{e}\right]^{2} \times\left[\mathrm{U}(1)_{m}\right]^{2}$ in the Zwanziger form. However, 
it does not mean the increase of the gauge degrees of freedom, because $\vec{A}_{\mu}$ and $\vec{B}_{\mu}$ are not independent fields due to the interaction between them in the Zwanziger form $(3.3)[34,35]$.

We phenomenologically introduce the QCD-monopoles as the relevant degrees of freedom to the color confinement in order to investigate the nonperturbative features in QCD. By introducing the QCD-monopole field $\chi_{\alpha}(\alpha=1,2,3)$ and its coupling with the dual gauge fields $\vec{B}_{\mu}$ [36], the effective Lagrangian is obtained by

$$
\begin{aligned}
\mathcal{L}_{\mathrm{DGL}} & =-\frac{1}{2 n^{2}}[n \cdot(\partial \wedge \vec{A})]^{\nu}[n \cdot *(\partial \wedge \vec{B})]_{\nu}+\frac{1}{2 n^{2}}[n \cdot(\partial \wedge \vec{B})]^{\nu}\left[n *^{*}(\partial \wedge \vec{A})\right]_{\nu} \\
& -\frac{1}{2 n^{2}}[n \cdot(\partial \wedge \vec{A})]^{2}-\frac{1}{2 n^{2}}[n \cdot(\partial \wedge \vec{B})]^{2} \\
& +\bar{q}(i \not \partial-e \vec{A} \cdot \vec{H}-m) q+\sum_{\alpha=1}^{3}\left[\left|\left(i \partial_{\mu}-g \vec{\epsilon}_{\alpha} \cdot \vec{B}_{\mu}\right) \chi_{\alpha}\right|^{2}-\lambda\left(\left|\chi_{\alpha}\right|^{2}-v^{2}\right)^{2}\right]
\end{aligned}
$$

where the self-interaction of $\chi_{\alpha}$ is introduced phenomenologically similar to the Ginzburg-Landau theory in the superconductivity physics [14,15]. We call the theory based on the Lagrangian (3.6) as the dual Ginzburg-Landau theory because it is a dual version of the Ginzburg-Landau theory.

In Eq.(3.6), $g$ is the unit magnetic charge of the magnetic monopoles, obeying the Dirac condition, $e g=4 \pi$. Let us consider the QCD-monopoles belonging to the fundamental representation of $\mathrm{SU}(3)_{c}$. In this case, the magnetic charge $\vec{\epsilon}_{\alpha}$ $(\alpha=1,2,3)$ of the QCD-monopole field $\chi_{\alpha}$ is found to be $\vec{\epsilon}_{1}=(1,0), \vec{\epsilon}_{2}=\left(-\frac{1}{2},-\frac{\sqrt{3}}{2}\right)$, and $\vec{\epsilon}_{3}=\left(-\frac{1}{2}, \frac{\sqrt{3}}{2}\right)$ in a suitable representation of $\mathrm{SU}(3)_{c}[14,15]$. In the Lagrangian (3.6), three kinds of the QCD-monopole fields $\chi_{\alpha}(\alpha=1,2,3)$ are complex fields, and there is a constraint among their phases, e.g. $\sum_{\alpha=1}^{3} \arg \chi_{\alpha}=0[14,15]$. One finds that the residual two degrees of freedom on the phase of $\chi_{\alpha}$ are closely related with the two kinds of the dual gauge field $\vec{B}^{\mu}=\left(B_{3}^{\mu}, B_{8}^{\mu}\right)$. The dual gauge symmetry $\left[\mathrm{U}(1)_{m}\right]^{2} \equiv \mathrm{U}(1)_{m}^{(3)} \times \mathrm{U}(1)_{m}^{(8)}$ in $(3.5)$ is connected to the local phase transformation of the QCD-monopole field,

$$
\begin{aligned}
& \vec{B}_{\mu}(x) \rightarrow \vec{B}_{\mu}(x)+\frac{1}{g} \partial_{\mu} \vec{\theta}_{B}(x), \\
& \chi_{\alpha}(x) \rightarrow e^{-i \vec{\epsilon}_{\alpha} \cdot \vec{\theta}_{B}(x)} \chi_{\alpha}(x)
\end{aligned}
$$

similar to the ordinary gauge symmetry $\left[\mathrm{U}(1)_{e}\right]^{2} \equiv \mathrm{U}(1)_{e}^{(3)} \times \mathrm{U}(1)_{e}^{(8)}$.

The dual Meissner effect is brought by the realization of QCD-monopole condensation due to the self-interaction of $\chi_{\alpha}$ with $v^{2}>0$ in the Lagrangian (3.6). 
QCD-monopole condensation leads the mass term of the dual gauge field $\vec{B}_{\mu}$, similar to the generation of the photon mass due to Cooper-pair condensation in the superconductivity. For the QCD vacuum, one finds $\left|\chi_{\alpha}\right|=v(\alpha=1,2,3)$ in the mean field level of the $\chi$-field, and therefore the Lagrangian (3.6) reads

$$
\begin{array}{r}
\mathcal{L}_{\mathrm{DGL}-\mathrm{MF}}=-\frac{1}{2 n^{2}}[n \cdot(\partial \wedge \vec{A})]^{\nu}\left[n *^{*}(\partial \wedge \vec{B})\right]_{\nu}+\frac{1}{2 n^{2}}[n \cdot(\partial \wedge \vec{B})]^{\nu}\left[n \cdot{ }^{*}(\partial \wedge \vec{A})\right]_{\nu} \\
-\frac{1}{2 n^{2}}[n \cdot(\partial \wedge \vec{A})]^{2}-\frac{1}{2 n^{2}}[n \cdot(\partial \wedge \vec{B})]^{2}+\bar{q}(i \not \partial-e \vec{A} \cdot \vec{H}-m) q+\frac{1}{2} m_{B}^{2} \vec{B}^{2}
\end{array}
$$

where $m_{B}=\sqrt{3} g v$ is the mass of the dual gauge field $\vec{B}_{\mu}$. The QCD-monopole field is also massive due to the self-interaction, $m_{\chi}=2 \sqrt{\lambda} v$. It is notable that the dual gauge symmetry $\left[\mathrm{U}(1)_{m}\right]^{2}$ in $(3.5)$ is broken, while there remains the gauge symmetry $\left[\mathrm{U}(1)_{e}\right]^{2}$, which is the subgroup of the original gauge group $\mathrm{SU}\left(N_{c}\right)$. Hence, QCD-monopole condensation never breaks the abelian gauge symmetry $\left[\mathrm{U}(1)_{e}\right]^{2}$, subgroup of $\mathrm{SU}(3)_{c}{ }^{\star}$

To investigate the QCD vacuum, it is useful to start with the mean-field level Lagrangian (3.8), neglecting the fluctuation of the QCD-monopole field. By integrating out the dual gauge field $\vec{B}_{\mu}$ in the partition functional, one gets the Lagrangian described by the gauge field $\vec{A}_{\mu}$,

$$
\mathcal{L}_{\mathrm{DGL}-\mathrm{MF}}=-\frac{1}{4} \vec{f}_{\mu \nu} \vec{f}^{\mu \nu}+\frac{1}{2} \vec{A}^{\mu} K_{\mu \nu} \vec{A}^{\nu}+\bar{q}(i \not \partial-e \vec{A} \cdot \vec{H}-m) q
$$

In this equation, the non-local operator $K^{\mu \nu}$ is defined by

$$
K^{\mu \nu} \equiv \frac{n^{2} m_{B}^{2}}{(n \cdot \partial)^{2}+n^{2} m_{B}^{2}} X^{\mu \nu}
$$

where $X^{\mu \nu}$ is given by

$$
\begin{aligned}
X^{\mu \nu} & \equiv \frac{1}{n^{2}} \epsilon_{\lambda}{ }^{\mu \alpha \beta} \epsilon^{\lambda \nu \gamma \delta} n_{\alpha} n_{\gamma} \partial_{\beta} \partial_{\delta} \\
& =\frac{1}{n^{2}}\left[-n^{2} \partial^{2} g^{\mu \nu}+(n \cdot \partial)^{2} g^{\mu \nu}+n^{\mu} n^{\nu} \partial^{2}-(n \cdot \partial)\left(n^{\mu} \partial^{\nu}+n^{\nu} \partial^{\mu}\right)+n^{2} \partial^{\mu} \partial^{\nu}\right]
\end{aligned}
$$

One easily finds the $\left[\mathrm{U}(1)_{e}\right]^{2}$-gauge invariance of the Lagrangian $\mathcal{L}_{\mathrm{DGL}-\mathrm{MF}}$ in

* QCD-monopole condensation may break the off-diagonal part of the original gauge symmetry $\mathrm{SU}(3)_{c}$. It is, however, less important because the off-diagonal part already has no gauge symmetry due to the abelian gauge fixing. 
Eq.(3.9) by using the relations,

$$
X_{\mu \nu}=X_{\nu \mu}, \quad X_{\mu \nu} \partial^{\nu}=X_{\mu \nu} n^{\nu}=0
$$

Hence, we find again that QCD-monopole condensation never breaks the gauge symmetry $\left[\mathrm{U}(1)_{e}\right]^{2} \in \mathrm{SU}(3)$. The $m_{B}$-dependent term in the effective Lagrangian (3.9) originally corresponds to the mass term of the dual gauge field $\vec{B}_{\mu}$, and leads to the dual Meissner effect or the color confinement.

We discuss the spatial distribution of the QCD-monopole condensate $\left|\chi_{\alpha}(x)\right|$ and the color-electric field $E(x)$ in the hadron flux tube within the quenched approximation [16]. We consider the flux tube in the mesonic system including the static quark and the antiquark with a relative distance $\mathbf{r}$. A straight flux tube is formed between the quark and the antiquark, and therefore the system is axial symmetric around the axis between them [16]. In a large separation limit, $|\mathbf{r}| \rightarrow \infty$, the system has a translational invariance along the direction $\hat{\mathbf{r}}$, and the system becomes quite similar to a vortex solution in the superconductivity [37] or the Nielsen-Olesen vortex in the abelian Higgs model [38].

In this subject, it is convenient to describe the dual Ginzburg-Landau Lagrangian (3.6) only by the $\vec{B}_{\mu}$ field,

$$
\mathcal{L}_{\mathrm{DGL}}=-\frac{1}{4}\left(\partial_{\mu} \vec{B}_{\nu}-\partial_{\nu} \vec{B}_{\mu}\right)^{2}+\sum_{\alpha=1}^{3}\left[\left|\left(i \partial_{\mu}-g \vec{\epsilon}_{\alpha} \cdot \vec{B}_{\mu}\right) \chi_{\alpha}\right|^{2}-\lambda\left(\left|\chi_{\alpha}\right|^{2}-v^{2}\right)^{2}\right]
$$

We investigate the solution of the coupled equation of the abelian monopole field $\left|\chi_{\alpha}(x)\right|$ and the $\vec{B}_{\mu}(x)$ field at the tree level, which is analogous to the vortex solution in the superconductivity [37]. The solutions for the color-electric field and the QCD-monopole field are given by functions of $\rho$, the distance from the cylindrical axis, and take forms as $E_{\operatorname{diag}}(\rho) \equiv E_{3}(\rho)=E_{8}(\rho)$ and $|\chi(\rho)| \equiv\left|\chi_{1}(\rho)\right|=$ $\left|\chi_{2}(\rho)\right|=\left|\chi_{3}(\rho)\right|$ in the flux tube [16].

We consider the case of $m_{\chi}>m_{B}$ corresponding to the type II superconductor because $m_{\chi}>m_{B}$ is suggested from the study of the quark potential as will be shown in the following chapter. The color-electric field $E_{\text {diag }}(\rho)$ takes a large value only in a region of $\rho \lesssim m_{B}^{-1}$, and therefore the cylindrical radius of the hadron flux tube is roughly given by $m_{B}^{-1}$. One finds the reduction of the QCD-monopole condensate $|\chi(\rho)|$ in the central region of $\rho \lesssim m_{\chi}^{-1}$ in the flux tube $[37,38]$. The QCD-monopole condensate is regarded as an almost constant value $v,|\chi(\rho)| \simeq v$, for the infrared region $\rho \gtrsim m_{\chi}^{-1}$ corresponding to $k_{T} \lesssim m_{\chi}$ in the momentum space, where $k_{T}$ denotes the transverse momentum component. On the contrary, the QCD-monopole condensate almost disappear, $|\chi(\rho)| \simeq 0$ for the ultraviolet 
region $\rho \lesssim m_{\chi}^{-1}$ corresponding to $k_{T} \gtrsim m_{\chi}$ in the momentum space. Thus, one finds the approximate relations,

$$
|\chi(\rho)| \simeq v \theta\left(\rho-m_{\chi}^{-1}\right) \quad \text { and } \quad m_{B}(\rho) \simeq \sqrt{3} g v \cdot \theta\left(\rho-m_{\chi}^{-1}\right),
$$

and therefore the ultraviolet cutoff appears in $m_{B}$. The appearance of the ultraviolet cutoff is also found in the argument of the vortex in the superconductivity [37], and is important for the study of the string tension as will be shown in the next chapter.

We have obtained a phenomenological theory of the nonperturbative QCD, the dual Ginzburg-Landau theory, which contains the confining mechanism through QCD-monopole condensation and the dual Meissner effect. By using the Lagrangian (3.6) or (3.9) in this theory, we investigate the nonperturbative features of QCD, the color confinement, the effect of the $q-\bar{q}$ pair creation, and the dynamical chiral-symmetry breaking.

\section{Quark Confinement Potential in the Quenched Approximation}

In this chapter, we derive the quark static potential in the dual GinzburgLandau theory, based on the Lagrangian (3.6) or (3.9) within the quenched approximation. The effective Lagrangian including the quark current $\vec{j}_{\mu}$ is given by

$$
\begin{aligned}
\mathcal{L}_{\mathrm{DGL}-\mathrm{MF}} & =\frac{1}{2} \vec{A}^{\mu} D_{\mu \nu}^{-1} \vec{A}^{\nu}+\vec{j}_{\mu} \vec{A}^{\mu} \\
& =\frac{1}{2}\left(\vec{A}^{\mu}+\vec{j}_{\alpha} D^{\alpha \mu}\right) D_{\mu \nu}^{-1}\left(\vec{A}^{\nu}+D^{\nu \beta} \vec{j}_{\beta}\right)-\frac{1}{2} \vec{j}_{\mu} D^{\mu \nu} \vec{j}_{\nu},
\end{aligned}
$$

where $D_{\mu \nu}$ is the propagator of the diagonal gluon field, $\vec{A}_{\mu}$. By integrating out $\vec{A}_{\mu}$ in the partition functional, one gets the quark-current correlation,

$$
\mathcal{L}_{j}=-\frac{1}{2} \vec{j}_{\mu} D^{\mu \nu} \vec{j}_{\nu}
$$

The diagonal-gluon propagator $D^{\mu \nu}$ is obtained from the effective Lagrangian (3.9) in the dual Ginzburg-Landau theory, and the inverse propagator of the diagonal gluon field is given as

$$
D_{\mu \nu}^{-1}=g_{\mu \nu} \partial^{2}-\left(1-\frac{1}{\alpha_{e}}\right) \partial_{\mu} \partial_{\nu}+\frac{m_{B}^{2} n^{2}}{(n \cdot \partial)^{2}+m_{B}^{2} n^{2}} X_{\mu \nu}
$$

in the Lorentz gauge with the gauge fixing term, $\mathcal{L}_{G . F .}=-\frac{1}{2 \alpha_{e}}\left(\partial_{\mu} \vec{A}^{\mu}\right)^{2}$. Then, the 
diagonal-gluon propagator is given by

$$
D_{\mu \nu}=\frac{1}{\partial^{2}}\left\{g_{\mu \nu}+\left(\alpha_{e}-1\right) \frac{\partial_{\mu} \partial_{\nu}}{\partial^{2}}\right\}-\frac{1}{\partial^{2}} \frac{m_{B}^{2}}{\partial^{2}+m_{B}^{2}} \frac{n^{2}}{(n \cdot \partial)^{2}} X_{\mu \nu}
$$

and therefore the quark-current correlation (4.2) leads

$$
\begin{aligned}
\mathcal{L}_{j} & =-\frac{1}{2} \vec{j}^{\mu}\left[\frac{1}{\partial^{2}} g_{\mu \nu}-\frac{1}{\partial^{2}} \frac{m_{B}^{2}}{\partial^{2}+m_{B}^{2}} \frac{n^{2}}{(n \cdot \partial)^{2}}\left\{-\partial^{2}\left(g_{\mu \nu}-\frac{n_{\mu} n_{\nu}}{n^{2}}\right)+\frac{(n \cdot \partial)^{2}}{n^{2}} g_{\mu \nu}\right\}\right] \vec{j}^{\nu} \\
& =-\frac{1}{2} \vec{j}^{\mu}\left[\frac{1}{\partial^{2}+m_{B}^{2}} g_{\mu \nu}+\frac{m_{B}^{2}}{\partial^{2}+m_{B}^{2}} \frac{n^{2}}{(n \cdot \partial)^{2}}\left(g_{\mu \nu}-\frac{n_{\mu} n_{\nu}}{n^{2}}\right)\right] \vec{j}^{\nu}
\end{aligned}
$$

independent of the gauge-fixing parameter $\alpha_{e}$. The action including the quarkcurrent correlation is given by

$$
\begin{aligned}
S_{j} & \equiv \int d^{4} x \mathcal{L}_{j} \\
& =\int \frac{d^{4} k}{(2 \pi)^{4}} \frac{1}{2} \vec{j}^{\mu}(-k)\left[\frac{1}{k^{2}-m_{B}^{2}} g_{\mu \nu}+\frac{-m_{B}^{2}}{k^{2}-m_{B}^{2}} \frac{n^{2}}{(n \cdot k)^{2}}\left(g_{\mu \nu}-\frac{n_{\mu} n_{\nu}}{n^{2}}\right)\right] \vec{j}^{\nu}(k),
\end{aligned}
$$

where $\vec{j}_{\mu}(k)$ is the Fourier component of $\vec{j}_{\mu}(x)$ with $\vec{j}_{\mu}(k) \equiv \int d^{4} x e^{i k \cdot x} \vec{j}_{\mu}(x)$.

Let us consider the static system of a heavy quark and antiquark pair with opposite color-charge located at $\mathbf{a}$ and $\mathbf{b}$, respectively [14,15]. In this case, the quark current is given by

$$
\vec{j}_{\mu}(x)=\vec{Q} g_{\mu 0}\left\{\delta^{3}(\mathbf{x}-\mathbf{b})-\delta^{3}(\mathbf{x}-\mathbf{a})\right\},
$$

where $\vec{Q}=\left(Q_{3}, Q_{8}\right)$ is the color charge of the static quark [39], and its Fourier component reads

$$
\vec{j}_{\mu}(k)=\vec{Q} g_{\mu 0} 2 \pi \delta\left(k_{0}\right)\left(e^{-i \mathbf{k} \cdot \mathbf{b}}-e^{-i \mathbf{k} \cdot \mathbf{a}}\right) .
$$

The action (4.6) reads

$$
S_{j}=-\vec{Q}^{2} \int d t \int \frac{d^{3} k}{(2 \pi)^{3}} \frac{1}{2}\left(1-e^{i \mathbf{k} \cdot \mathbf{r}}\right)\left(1-e^{-i \mathbf{k} \cdot \mathbf{r}}\right)\left[\frac{1}{\mathbf{k}^{2}+m_{B}^{2}}+\frac{m_{B}^{2}}{\mathbf{k}^{2}+m_{B}^{2}} \cdot \frac{1}{(\mathbf{n} \cdot \mathbf{k})^{2}}\right],
$$

where $\mathbf{n}$ is a unit vector and $\mathbf{r} \equiv \mathbf{b}-\mathbf{a}$ is the relative distance between the quark and the antiquark. The static quark potential thus obtained is divided into two 
parts,

$$
V(\mathbf{r} ; \mathbf{n})=V_{\text {Yukawa }}(r)+V_{\text {linear }}(\mathbf{r} ; \mathbf{n}) .
$$

Here, $V_{\text {Yukawa }}(r)$ gives the Yukawa-type potential,

$$
\begin{aligned}
V_{\text {Yukawa }}(r) & \equiv \vec{Q}^{2} \int \frac{d^{3} k}{(2 \pi)^{3}} \frac{1}{2}\left(1-e^{i \mathbf{k} \cdot \mathbf{r}}\right)\left(1-e^{-i \mathbf{k} \cdot \mathbf{r}}\right) \frac{1}{\mathbf{k}^{2}+m_{B}^{2}} \\
& =-\vec{Q}^{2} \int \frac{d^{3} k}{(2 \pi)^{3}} e^{i \mathbf{k} \cdot \mathbf{r}} \frac{1}{\mathbf{k}^{2}+m_{B}^{2}}=-\frac{\vec{Q}^{2}}{4 \pi} \cdot \frac{e^{-m_{B} r}}{r}
\end{aligned}
$$

apart from a irrelevant constant. $V_{\text {linear }}(\mathbf{r} ; \mathbf{n})$ is given by

$$
\begin{aligned}
V_{\text {linear }}(\mathbf{r} ; \mathbf{n}) & \equiv \vec{Q}^{2} \int \frac{d^{3} k}{(2 \pi)^{3}} \frac{1}{2}\left(1-e^{i \mathbf{k} \cdot \mathbf{r}}\right)\left(1-e^{-i \mathbf{k} \cdot \mathbf{r}}\right) \frac{m_{B}^{2}}{\mathbf{k}^{2}+m_{B}^{2}} \cdot \frac{1}{(\mathbf{n} \cdot \mathbf{k})^{2}} \\
& =\vec{Q}^{2} \int \frac{d^{3} k}{(2 \pi)^{3}}\{1-\cos (\mathbf{k} \cdot \mathbf{r})\} \frac{m_{B}^{2}}{\mathbf{k}^{2}+m_{B}^{2}} \cdot \frac{1}{(\mathbf{n} \cdot \mathbf{k})^{2}}
\end{aligned}
$$

Because of the axial symmetry of the system, it is reasonable to take $\mathbf{n} / / \mathbf{r}$, and therefore one finds

$$
\begin{aligned}
V_{\text {linear }}(r) & =\vec{Q}^{2} \int_{-\infty}^{\infty} \frac{d k_{r}}{2 \pi} \int \frac{d^{2} k_{T}}{(2 \pi)^{2}}\left\{1-\cos \left(k_{r} r\right)\right\} \frac{m_{B}^{2}}{k_{r}^{2}+k_{T}^{2}+m_{B}^{2}} \cdot \frac{1}{k_{r}^{2}} \\
& =\frac{\vec{Q}^{2} m_{B}^{2}}{8 \pi^{2}} \int_{-\infty}^{\infty} \frac{d k_{r}}{k_{r}^{2}}\left\{1-\cos \left(k_{r} r\right)\right\} \int_{0}^{\infty} d k_{T}^{2} \frac{1}{k_{r}^{2}+k_{T}^{2}+m_{B}^{2}}
\end{aligned}
$$

where $k_{T}$ denotes the momentum component perpendicular to $\mathbf{r}$.

It should be noted that there appears a physical ultraviolet cutoff in the $k_{T^{-}}$ integral corresponding to Eq.(3.14), and therefore no ultraviolet divergence comes in Eq.(4.13). The appearance of the physical ultraviolet cutoff is similar to the argument of the vortex in the superconductivity [37] as mentioned in the previous chapter. In the central region of the hadron flux tube, $\rho \lesssim m_{\chi}^{-1}$, one find $m_{B} \simeq 0$ because the QCD-monopole field $|\chi|$ almost vanishes. Thus, one gets Eq.(3.14), and there appears the ultraviolet cutoff, $k_{T} \lesssim m_{\chi}$, in the integral in Eq.(4.13) similar to the argument of the vortex in the superconductivity [37].

* In terms of the energy minimum condition in Eq.(4.12), one gets $\mathbf{n} / / \mathbf{r}$. Otherwise, there appears the infrared divergence, corresponding to the double pole in $V_{\text {linear }}$. 
Hence, we obtain the linear potential,

$$
\begin{aligned}
V_{\text {linear }}(r) & =\frac{\vec{Q}^{2} m_{B}^{2}}{8 \pi^{2}} \int_{-\infty}^{\infty} \frac{d k_{r}}{k_{r}^{2}}\left\{1-\cos \left(k_{r} r\right)\right\} \ln \left(\frac{m_{\chi}^{2}+k_{r}^{2}+m_{B}^{2}}{k_{r}^{2}+m_{B}^{2}}\right) \\
& =\operatorname{Re} \frac{\vec{Q}^{2} m_{B}^{2}}{8 \pi^{2}} \int_{-\infty}^{\infty} \frac{d k_{r}}{k_{r}^{2}}\left(1-e^{i k_{r} r}\right) \ln \left(\frac{m_{\chi}^{2}+k_{r}^{2}+m_{B}^{2}}{k_{r}^{2}+m_{B}^{2}}\right) \\
& =\left.\operatorname{Re} \frac{\vec{Q}^{2} m_{B}^{2}}{8 \pi^{2}} \pi i \frac{1-e^{i k_{r} r}}{k_{r}} \ln \left(\frac{m_{\chi}^{2}+k_{r}^{2}+m_{B}^{2}}{k_{r}^{2}+m_{B}^{2}}\right)\right|_{k_{r}=0} \\
& =\frac{\vec{Q}^{2} m_{B}^{2}}{8 \pi} \ln \left(\frac{m_{B}^{2}+m_{\chi}^{2}}{m_{B}^{2}}\right) \cdot r
\end{aligned}
$$

where we have used the orthodox technique of the complex integration. The final expression of the quark static potential is given by

$$
V(r)=-\frac{\vec{Q}^{2}}{4 \pi} \frac{e^{-m_{B} r}}{r}+\frac{\vec{Q}^{2} m_{B}^{2}}{8 \pi} \ln \left(\frac{m_{B}^{2}+m_{\chi}^{2}}{m_{B}^{2}}\right) \cdot r,
$$

where $\vec{Q}^{2}$ takes the same value for the red, blue and green quarks, $\vec{Q}^{2}=Q_{3}^{2}+Q_{8}^{2}=$ $\frac{e^{2}}{3}[39]$. We obtain a simple formula for the string tension,

$$
k=\frac{\vec{Q}^{2} m_{B}^{2}}{8 \pi} \ln \left(\frac{m_{B}^{2}+m_{\chi}^{2}}{m_{B}^{2}}\right)
$$

which is analogous to the energy per unit length of the vortex in a type-II superconductor,

$$
\epsilon=\left(\frac{\phi_{0}}{4 \pi \delta}\right)^{2} \ln (\delta / \xi)=\frac{\phi_{0}^{2} m_{A}^{2}}{32 \pi^{2}} \ln \left(\frac{m_{\phi}^{2}}{m_{A}^{2}}\right),
$$

where $\phi_{0}$ is a magnetic flux of the vortex, $\delta=m_{A}^{-1}$ and $\xi=m_{\phi}^{-1}$ denote the penetration depth and the coherent length, respectively [10,37].

It is worth mentioning that our expression for the quark confining potential Eq.(4.15) differs from the result of the Kanazawa group [14,15]. Their analytic expression for the quark potential includes three parts, and looks rather complicated. (See Eq.(4.9) in Ref.15.) Moreover, their expression for the string tension is given by the modified Bessel function, $K_{0}(x)$, and differs from our expression, 
Eq.(4.16). Such difference stems on the usage of the following "prescription" by the Kanazawa group, which used an artificial replacement as

$$
\frac{1}{(n \cdot k)^{2}} \rightarrow \frac{1}{2}\left[\frac{1}{(n \cdot k+i a)^{2}}+\frac{1}{(n \cdot k-i a)^{2}}\right]
$$

to avoid the infrared double pole in the gluon propagator $[15,40]$, where $a$ cannot be taken to the zero limit. However, this replacement seems troublesome and gives several difficulties as will be shown in chapter 6. (See Eq.(6.1).) Moreover, there does not appear the infrared double pole in the expression of the quark potential in Eq.(4.13), owing to the axial symmetry of the system, $\mathbf{n} / / \mathbf{r}$. Hence, there is no need to eliminate the "double pole" in this subject, which is similar to the vortex in the superconductivity: there does not appear the unphysical divergence related to the infrared double pole in the vortex solution. The replacement (4.18) of the Kanazawa group is unnecessary to obtain the quark potential.

We compare the static quark potential, Eq.(4.15), with the phenomenological potential, for instance, the Cornell potential [41] in Fig.1. The quark potential is thus reproduced in our theory by choosing $e=5.5$ (the gauge coupling constant), $v=126 \mathrm{MeV}$ and $\lambda=25$ in Lagrangian (3.6), which provide $g=2.3, m_{B}=500 \mathrm{MeV}$ (mass of the dual gauge field $\vec{B}_{\mu}$ ) and $m_{\chi}=1.26 \mathrm{GeV}$ (QCD-monopole mass). This parameter set gives $k=1.0 \mathrm{GeV} / \mathrm{fm}$ for the string tension in Eq.(4.16), and the radius of the hadron flux tube $m_{B}^{-1} \simeq 0.4 \mathrm{fm}$. The linear part of the quark potential is responsible for the quark confinement and characterizes the nonperturbative feature in the infrared region.

We discuss now the nonperturbative effect on the quark potential in the relatively short distance, $r \sim 0.2 \mathrm{fm} \simeq(1 \mathrm{GeV})^{-1}$, by comparing the results of the dual Ginzburg-Landau theory and the Cornell potential. The Cornell potential has the Coulomb part and the linear part,

$$
V_{\text {Cornell }}(r)=-\frac{e_{C}^{2}}{3 \pi} \cdot \frac{1}{r}+k_{C} r,
$$

where the parameters, $e_{C} \simeq 2$ and $k_{C} \simeq 1 \mathrm{GeV} / \mathrm{fm}$, are chosen to reproduce the lattice QCD data in the pure gauge case and several data of the heavy quarkonium [41]. In the Cornell potential, $k_{C}$ in the linear part is nothing but the string tension $k$. On the other hand, the Coulomb part is introduced to represent the perturbative one-gluon exchange effect, and therefore $e_{C}$ seems to correspond to the gauge coupling constant. Surely, the Coulomb potential obtained from the one-gluon exchange would be valid for quite a short distance as $r \ll 0.2 \mathrm{fm}$. In the region of $r \sim 0.2 \mathrm{fm} \simeq(1 \mathrm{GeV})^{-1}$, there remains the strong coupling, $\alpha_{S} \sim 0.5$, 
so that the perturbative theory should not be workable and there must be some contribution beyond the one-gluon exchange effect. In this respect, $e_{C}$ does not correspond to the gauge coupling constant of QCD directly, but should be regarded as a fitting parameter including the higher-order effect of the perturbation or the nonperturbative effect.

There should be a nonperturbative effect even in the region of $r \sim 0.2 \mathrm{fm} \simeq$ $(1 \mathrm{GeV})^{-1}$ in the dual Ginzburg-Landau theory, because of the presence of QCDmonopole condensation, whose energy scale is characterized by $m_{\chi}$. This nonperturbative effect is found as a reduction of the effective degrees of freedom of gluons due to QCD-monopole condensation, because the off-diagonal gluons are considered to lose their activities in this infrared region. Although there are $N_{c}^{2}-1$ gluons in the $\mathrm{SU}\left(N_{c}\right)$ nonabelian gauge theory, only $N_{c}-1$ diagonal gluon fields are considered to have a large contribution to the infrared region in the dual GinzburgLandau theory. Hence, the color charge seems to be reduced to the effective charge in the one-gluon exchange form,

$$
e_{\mathrm{eff}}^{2}=\frac{\sum_{\beta \in \operatorname{diag}}\left(Q_{\beta}^{2}\right) e^{2}}{\sum_{\alpha}\left(Q_{\alpha}^{2}\right)}=\frac{\left(N_{c}-1\right) e^{2}}{N_{c}^{2}-1}=\frac{e^{2}}{N_{c}+1},
$$

where $\sum_{\beta \in \text { diag }}$ denotes the summation over the diagonal component alone. Then, the parameter $e_{C}$ in the Coulomb part of the Cornell potential in Eq.(4.19) should correspond to $e_{\text {eff }}=\frac{e}{2}$ in the dual Ginzburg-Landau theory with the $N_{c}=3$ case. This relation is also obtained by the formal comparison between the Yukawa part in Eq.(4.15) and the Coulomb part in Eq.(4.19) at a short distance.

The derivation of the quark potential (4.15) is based on the quenched approximation, where we have neglected the dynamics of the quark field. However, in the real QCD system, effects of the dynamical quark should be important in the infrared (long-range) region of the potential, because the dynamical $q-\bar{q}$ pair creation should take place as the hadronic string becomes longer than a critical value. Due to the $q-\bar{q}$ pair-creation effect, the linear potential between quark and antiquark is screened in the infrared (long-distance) region, and the quark static potential should be asymptotically saturated. Such an effect has been observed in the lattice QCD simulation with dynamical quarks [24]. In the next chapter, we study the $q-\bar{q}$ pair creation phenomena and its effect on the quark confining potential. 


\section{The $q-\bar{q}$ Pair Creation, Infrared Screening Effect on the Quark Confinement Potential and Infrared Cutoff in the Gluon Propagator}

In this chapter, we study the dynamical effect of the light quarks, particularly the infrared screening on the quark potential due to the dynamical $q-\bar{q}$ pair creation in the hadron flux tube. In the real strong interaction system, there are two or three kinds of light quarks ( $\mathrm{u}, \mathrm{d}$ and s-quark). The introduction of such light dynamical quarks is important not only for the dynamical chiral-symmetry breaking [4], but also for the color confinement, because the quark confining potential is screened in the infrared region [24] due to the $q-\bar{q}$ pair creation [29].

In order to obtain the $q-\bar{q}$ pair creation rate, we introduce the dynamical quark field interacting with the color-electric field, which is approximated as a homogeneous external field inside the hadron flux tube. The Lagrangian including the quark and the external abelian gluons, $\vec{A}^{\mu}=\left(A_{3}^{\mu}, A_{8}^{\mu}\right)$, is given by

$$
\mathcal{L}=\bar{q}(i \not \partial-e / A-M) q
$$

where $M$ is the effective quark mass, and $A_{\mu} \equiv \vec{A}_{\mu} \cdot \vec{H}$. By integrating out the quark field in the partition functional, we obtain the effective action [42],

$$
\begin{aligned}
S_{\mathrm{eff}} & =-\frac{i}{2} \operatorname{Tr} \ln \left\{-\left(i \partial_{\mu}-e A_{\mu}\right)^{2}-\frac{e}{2} \sigma_{\mu \nu} f^{\mu \nu}+M^{2}-i \epsilon\right\} \\
& =\frac{i}{2} \int d^{4} x \int_{0}^{\infty} \frac{d \tau}{\tau} \operatorname{tr}\left\langle x\left|\exp \left[-i \tau\left\{-\left(i \partial_{\mu}-e A_{\mu}\right)^{2}-\frac{e}{2} \sigma_{\mu \nu} f^{\mu \nu}+M^{2}-i \epsilon\right\}\right]\right| x\right\rangle
\end{aligned}
$$

with $\sigma_{\mu \nu} \equiv \frac{1}{2 i}\left[\gamma_{\mu}, \gamma_{\nu}\right]$ and $f_{\mu \nu} \equiv \partial_{\mu} A_{\nu}-\partial_{\nu} A_{\mu}$. After some calculation [29], the effective Lagrangian is given by

$$
\mathcal{L}_{\text {eff }}=\frac{i N_{f}}{2 \pi} \int_{0}^{\infty} \frac{d \tau}{\tau} e^{-i \tau\left(M^{2}-i \epsilon\right)} \int \frac{d^{2} p_{T}}{(2 \pi)^{2}} e^{-i \tau p_{T}^{2}} \operatorname{tr}_{c}(e E) \operatorname{coth}(e E \tau) .
$$

Here, $E$ is a diagonal matrix with the color indices corresponding to the magnitude of the color-electric field, $E=\left\{\left(E_{3} T_{3}+E_{8} T_{8}\right)^{2}\right\}^{1 / 2}$, where $E_{3}=\left(E_{3}^{i} E_{3}^{i}\right)^{1 / 2}$ and $E_{8}=\left(E_{8}^{i} E_{8}^{i}\right)^{1 / 2}$ are the magnitude of the two kinds of color-electric field, $\vec{E}^{i}=$ $\left(E_{3}^{i}, E_{8}^{i}\right)=\partial^{0} \vec{A}^{i}-\partial^{i} \vec{A}^{0}(i=1,2,3)$. The integration variable $p_{T}$ physically means the momentum component of dynamical quarks perpendicular to the direction of the color-electric field. By using the Wick rotation in the complex $\tau$-plane, one 
obtains the Schwinger formula for the $q-\bar{q}$ pair creation rate $w$ [29],

$$
\begin{aligned}
w & =2 \operatorname{Im} \mathcal{L}_{\mathrm{eff}}=\frac{N_{f}}{\pi} \sum_{l=1}^{\infty} \int \frac{d^{2} p_{T}}{(2 \pi)^{2}} \frac{1}{l} \operatorname{tr}_{c}(e E) e^{-l \pi\left(p_{T}^{2}+M^{2}\right)(e E)^{-1}} \\
& =-\frac{N_{f}}{2 \pi^{2}} \int_{0}^{\infty} d p_{T} p_{T} \operatorname{tr}_{c}(e E) \ln \left\{1-e^{-\pi\left(p_{T}^{2}+M^{2}\right)(e E)^{-1}}\right\} \\
& =\frac{N_{f}}{4 \pi^{3}} \sum_{l=1}^{\infty} \frac{1}{l^{2}} \operatorname{tr}_{c}(e E)^{2} e^{-l \pi M^{2}(e E)^{-1}}
\end{aligned}
$$

which is purely a nonperturbative effect in terms of the gauge coupling constant $e$, and cannot be obtained by the use of the perturbation with respect to $e$.

Let us consider the expression of $e E$ in the hadron flux tube with the cross section $S, S \sim \pi m_{B}^{-2} \sim 0.5 \mathrm{fm}^{2}$. In a fundamental representation of $\mathrm{SU}(3)_{c}$, the red $(R)$, blue $(B)$ and green $(G)$ quarks are expressed by $(1,0,0),(0,1,0),(0,0,1)$, respectively. In this representation, the color charge $\vec{Q}$ is given by $\left(Q_{3}, Q_{8}\right)=$ $\left(\frac{1}{2} e, \frac{1}{2 \sqrt{3}} e\right),\left(-\frac{1}{2} e, \frac{1}{2 \sqrt{3}} e\right),\left(0,-\frac{1}{\sqrt{3}} e\right)$ for the red, blue and green quark, respectively [39]. Then, the diagonal matrix $e E$, which corresponds to the magnitude of the color-electric field, is given by

$$
e E=e\left\{\left(E_{3} T_{3}+E_{8} T_{8}\right)^{2}\right\}^{1 / 2}=\frac{e}{S}\left\{\left(Q_{3} T_{3}+Q_{8} T_{8}\right)^{2}\right\}^{1 / 2}=\operatorname{diag}(2 k, k, k)
$$

in the flux tube of the $R-\bar{R}$ system. Here, we have used the relation on the string tension, $k=\frac{\vec{Q}^{2}}{2 S}=\frac{e^{2}}{6 S}(\simeq 1 \mathrm{GeV} / \mathrm{fm})$, which is derived from the Gauss law $\vec{Q}=\vec{E} S$, and $k=\frac{1}{2} \vec{E}^{2} S=\frac{1}{2}\left(E_{3}^{2}+E_{8}^{2}\right) S$. ${ }^{\star}$ Similarly, one finds $e E=$ $\operatorname{diag}(k, 2 k, k), \operatorname{diag}(k, k, 2 k)$ for the $B-\bar{B}$ and $G-\bar{G}$ system, respectively.

Hence, one finds the same expression for $w$ in the $R-\bar{R}, B-\bar{B}$ and $G-\bar{G}$ system,

$$
\begin{aligned}
w & =-\frac{N_{f}}{2 \pi^{2}} \int_{0}^{\infty} d p_{T} p_{T}\left[2 k \ln \left\{1-e^{-\pi\left(p_{T}^{2}+M^{2}\right) /(2 k)}\right\}+2 \cdot k \ln \left\{1-e^{-\pi\left(p_{T}^{2}+M^{2}\right) / k}\right\}\right] \\
& =\frac{N_{f}}{4 \pi^{3}} \sum_{l=1}^{\infty} \frac{1}{l^{2}}\left[(2 k)^{2} e^{-l \pi M^{2} /(2 k)}+2 \cdot k^{2} e^{-l \pi M^{2} / k}\right] .
\end{aligned}
$$

The first term in the bracket corresponds to the $q-\bar{q}$ pair creation with the same color as the valence quark, and such a pair-creation process contributes to the cut

* Here, the spatial variation of the QCD-monopole field is neglected because it only appears in the central region of the flux tube, $\rho \lesssim m_{\chi}^{-1}$. 
of the hadron flux tube, i.e. the screening effect of the linear potential between the valence quarks. As Glendenning and Matsui [43] pointed out, the effective color charge of the created $q-\bar{q}$ pair becomes a half value due to the screening effect or the final-state interaction in this process, and $2 k$ in the first term of Eq.(5.6) is reduced to $k$. On the other hand, the second term in Eq.(5.6) describes the case that the color of the created $q-\bar{q}$ pair differs from that of the valence quark, so that this contribution is less important to the screening of the quark potential. Thus, the $q-\bar{q}$ pair creation rate relevant to the screening effect is given by

$$
w_{\mathrm{sc}}=-\frac{N_{f}}{2 \pi^{2}} k \int_{0}^{\infty} d p_{T} p_{T} \ln \left\{1-e^{-\pi\left(p_{T}^{2}+M^{2}\right) / k}\right\}=\frac{N_{f}}{4 \pi^{3}} k^{2} \sum_{l=1}^{\infty} \frac{1}{l^{2}} e^{-l \pi M^{2} / k}
$$

We estimate now the expectation value of the energy of the created $q-\bar{q}$ pair system. The creation rate of the $q-\bar{q}$ pair with the transverse momentum $p_{T}$ is given by

$$
w\left(p_{T}\right)=-\frac{N_{f}}{2 \pi^{2}} k p_{T} \ln \left\{1-e^{-\pi\left(p_{T}^{2}+M^{2}\right) / k}\right\},
$$

and therefore the expectation value of the created-pair energy is estimated by

$$
\left\langle 2 E_{q}\right\rangle=\frac{1}{w_{\mathrm{sc}}} \int_{0}^{\infty} d p_{T} w\left(p_{T}\right) \cdot 2 E_{q}\left(p_{T}\right)
$$

where $E_{q}\left(p_{T}\right)$ denotes the energy of a quark with the transverse momentum $p_{T}$ and is estimated as $E_{q}\left(p_{T}\right) \simeq\left(p_{T}^{2}+M^{2}\right)^{1 / 2 \dagger}$.

We show in Fig. 2 the $q-\bar{q}$ pair creation rate vs. the created-pair energy $2 E_{q}$. Here, we have used $k=1.0 \mathrm{GeV} / \mathrm{fm}$ and $M=350 \mathrm{MeV}$ for the string tension and the effective mass of the quark, respectively. We obtain the expectation value of the created pair energy, $\left\langle 2 E_{q}\right\rangle \simeq 850 \mathrm{MeV}$. Since the created pair energy $\left\langle 2 E_{q}\right\rangle$ is supplied by the shortening of the hadronic string by cutting off the string, the screening effect due to the $q-\bar{q}$ pair creation appears for the longer distance than $R_{\mathrm{sc}}$ such that $k R_{\mathrm{sc}} \simeq\left\langle 2 E_{q}\right\rangle$. Then, we obtain the screening distance $R_{\mathrm{sc}} \simeq 1 \mathrm{fm}$.

$\dagger$ Since $p_{T}^{2}=p_{x}^{2}+p_{y}^{2}$, it may be reasonable to use $E_{q}\left(p_{T}\right) \simeq\left(p^{2}+M^{2}\right)^{1 / 2}=\left(p_{x}^{2}+p_{y}^{2}+p_{z}^{2}+\right.$ $\left.M^{2}\right)^{1 / 2} \simeq\left(\frac{3}{2} p_{T}^{2}+M^{2}\right)^{1 / 2}$. However, the peak or the average of the $q-\bar{q}$ pair creation rate $w\left(p_{T}\right)$ is modified only slightly in this case.

$\ddagger$ The quark effective mass $M \simeq 350 \mathrm{MeV}$ is obtained in the low-momentum region by using the Schwinger-Dyson equation in our framework as will be shown in the next chapter. 
The hadronic strings become unstable against the $q-\bar{q}$ pair creation when the distance between the valence quark and antiquark becomes larger than $R_{\mathrm{sc}}$. This means the vanishing of the strong correlation between the quark and the antiquark in the infrared region due to the screening effect brought by the light $q-\bar{q}$ pair creation and the cut of the hadronic string. Then, the corresponding infrared cutoff, $\epsilon \simeq R_{\mathrm{sc}}^{-1} \simeq 200 \mathrm{MeV}$, should be introduced to the long-range strong correlation between the valence quarks.

In the dual Ginzburg-Landau theory, the long range correlation between quarks is brought by the nonlocal operator $\frac{1}{(n \cdot \partial)^{2}}$ in the diagonal-gluon propagator in Eq.(4.4). From the one-dimensional relation $\partial \frac{1}{\partial}(x)=\partial \theta(x)=\delta(x)$, one finds that $\frac{1}{\partial}(x)=\theta(x)+C_{1}$ with a constant $C_{1}$, and similarly one obtains

$$
\frac{1}{\partial^{2}}(x)=x \theta(x)+C_{1} x+C_{2},
$$

where $C_{1}$ and $C_{2}$ are arbitrary constants. Thus, one finds that

$$
\begin{aligned}
\frac{1}{(n \cdot \partial)^{2}}(x-y) & \equiv\left\langle x\left|\frac{1}{(n \cdot \partial)^{2}}\right| y\right\rangle \\
& =\left\{\left(x_{n}-y_{n}\right) \theta\left(x_{n}-y_{n}\right)+C_{1}\left(x_{n}-y_{n}\right)+C_{2}\right\} \delta^{3}\left(x_{T}-y_{T}\right)
\end{aligned}
$$

which gives a larger correlation for a further point to the direction $n_{\mu}$. Such an operator brings the strong long-range correlation along $n_{\mu}$, direction of the Dirac string, and leads to the linear confining potential in the absence of the dynamical quarks.

In the presence of light dynamical quarks, the infrared cutoff $\epsilon \simeq 200 \mathrm{MeV}$ should be introduced to this long-range correlation factor, $\frac{1}{(n \cdot \partial)^{2}}$, in the gluon propagator, in accordance with the $q-\bar{q}$ pair creation and the cut of the hadronic string. We get a modified diagonal-gluon propagator including the infrared screening effect as

$$
D_{\mu \nu}^{\mathrm{sc}}(k)=-\frac{1}{k^{2}}\left\{g_{\mu \nu}+\left(\alpha_{e}-1\right) \frac{k_{\mu} k_{\nu}}{k^{2}}\right\}+\frac{1}{k^{2}} \cdot \frac{m_{B}^{2}}{k^{2}-m_{B}^{2}} \cdot \frac{n^{2}}{(n \cdot k)^{2}+\epsilon^{2}} X_{\mu \nu},
$$

in the momentum representation from Eq.(4.4). Hence, the corresponding inverse

$\S$ In principle, the infrared screening effect in the gluon propagator would be calculable by solving the Schwinger-Dyson equation including the polarization effect of dynamical quarks.

I Although there is another way to introduce the infrared cutoff $\epsilon$, they qualitatively give the same results on the infrared screening effect in the quark potential. 
propagator is given by

$$
D_{\mu \nu}^{\mathrm{sc}}-1(k)=-\left\{g_{\mu \nu} k^{2}-\left(1-\frac{1}{\alpha_{e}}\right) k_{\mu} k_{\nu}\right\}-\frac{k^{2} m_{B}^{2} n^{2}}{k^{2}\left\{(n \cdot k)^{2}-m_{B}^{2} n^{2}\right\}+\epsilon^{2}\left(k^{2}-m_{B}^{2}\right)} X_{\mu \nu},
$$

and the modified Lagrangian reads

$$
\mathcal{L}_{\mathrm{DGL}-\mathrm{MF}}^{\mathrm{sc}}=\frac{1}{2} \vec{A}^{\mu} D_{\mu \nu}^{\mathrm{sc}}-1 \vec{A}^{\nu}+\bar{q}(i \not \partial-e \vec{A} \cdot \vec{H}-m) q,
$$

which holds the $[\mathrm{U}(1)]_{e}^{2}$-gauge invariance when the gauge fixing term is absent, i.e. $\alpha_{e}=\infty$. It is notable that the infrared double pole, e.g. $\frac{1}{(n \cdot k)^{2}}$, naturally disappears in the gluon propagator $D_{\mu \nu}^{\mathrm{sc}}(k)$, which comes to play an important role to the study of the dynamical chiral-symmetry breaking as will be shown in the chapter 6 . Then, the quark potential $V_{\text {linear }}(r)$ in Eq.(4.14) is modified by

$$
\begin{aligned}
V_{\text {linear }}^{\mathrm{sc}}(r) & \simeq \frac{\vec{Q}^{2} m_{B}^{2}}{8 \pi^{2}} \int_{-\infty}^{\infty} \frac{d k_{r}}{\left(k_{r}^{2}+\epsilon^{2}\right)}\left\{1-\cos \left(k_{r} r\right)\right\} \ln \left(\frac{m_{\chi}^{2}+k_{r}^{2}+m_{B}^{2}}{k_{r}^{2}+m_{B}^{2}}\right) \\
& =\operatorname{Re} \frac{\vec{Q}^{2} m_{B}^{2}}{8 \pi^{2}} \int_{-\infty}^{\infty} \frac{d k_{r}}{\left(k_{r}^{2}+\epsilon^{2}\right)}\left(1-e^{i k_{r} r}\right) \ln \left(\frac{m_{\chi}^{2}+k_{r}^{2}+m_{B}^{2}}{k_{r}^{2}+m_{B}^{2}}\right) \\
& =\left.\operatorname{Re} \frac{\vec{Q}^{2} m_{B}^{2}}{8 \pi^{2}} 2 \pi i \frac{1-e^{i k_{r} r}}{k_{r}+i \epsilon} \ln \left(\frac{m_{\chi}^{2}+k_{r}^{2}+m_{B}^{2}}{k_{r}^{2}+m_{B}^{2}}\right)\right|_{k_{r}=i \epsilon} \\
& =\frac{\vec{Q}^{2} m_{B}^{2}}{8 \pi} \cdot \frac{1-e^{-\epsilon r}}{\epsilon} \ln \left(\frac{m_{B}^{2}+m_{\chi}^{2}-\epsilon^{2}}{m_{B}^{2}-\epsilon^{2}}\right)
\end{aligned}
$$

apart from a small contribution of $O\left(\epsilon^{2}\right)$.

We obtain a compact formula for the quark potential including the infrared screening effect due to the $q-\bar{q}$ pair creation,

$$
V_{\mathrm{sc}}(r)=V_{\text {Yukawa }}(r)+V_{\text {linear }}^{\mathrm{sc}}(r)=-\frac{\vec{Q}^{2}}{4 \pi} \cdot \frac{e^{-m_{B} r}}{r}+k \cdot \frac{1-e^{-\epsilon r}}{\epsilon},
$$

where $k$ corresponds to the string tension,

$$
k=\frac{\vec{Q}^{2}}{8 \pi}\left[\left(m_{B}^{2}-\epsilon^{2}\right) \ln \left(\frac{m_{B}^{2}+m_{\chi}^{2}-\epsilon^{2}}{m_{B}^{2}-\epsilon^{2}}\right)+\epsilon^{2} \ln \left(\frac{m_{\chi}^{2}-\epsilon^{2}}{\epsilon^{2}}\right)\right] .
$$

The quark potential $V_{\text {linear }}^{\mathrm{sc}}(r)$ behaves as the linear potential with the string tension 
$k$,

$$
V_{\text {linear }}^{\text {sc }}(r) \simeq k r
$$

at the short distance, $r \ll \epsilon^{-1}$. On the contrary, the quark potential shows a saturation corresponding to the screening effect,

$$
V_{\text {linear }}^{\text {sc }}(r) \simeq \frac{k}{\epsilon}=\text { constant }
$$

in the infrared region, $r \gg \epsilon^{-1}$.

We show the numerical result of $V_{\text {linear }}^{\text {sc }}(r)$ for several values of infrared cutoff $\epsilon$ in Fig. 3 as the screening effect on the quark confining potential. We have used the same values for the gauge coupling constant and the mass of the dual gauge field as before, $e=5.5$ and $m_{B}=500 \mathrm{MeV}$. The QCD-monopole mass $m_{\chi}$ is chosen so as to reproduce the string tension $k=1.0 \mathrm{GeV} / \mathrm{fm}$, and is found to be $m_{\chi}=1.26$, 1.15 and $1.01 \mathrm{GeV}$ for $\epsilon=0,100$ and $200 \mathrm{MeV}$, respectively. The screening effect or the saturation of the quark confining potential is found in the long distance, and such a screening effect becomes dominant as $\epsilon$ gets larger.

Let us consider the relationship among the quantities related to the quark confinement and the infrared screening effect. As for the infrared cutoff $\epsilon$ and the effective quark mass $M$, we find a simple relation

$$
\epsilon \simeq \frac{k}{\left\langle 2 E_{q}\right\rangle} \sim \frac{k}{2 M}
$$

from the relations, $\epsilon \simeq R_{\mathrm{sc}}^{-1}$ and $k R_{\mathrm{sc}} \simeq\left\langle 2 E_{q}\right\rangle \sim 2 M$. We also find a simple relation between the saturated value of the confining potential, $V_{\text {linear }}^{\text {sc }}(\infty)$, and the string tension $k$,

$$
V_{\text {linear }}^{\text {sc }}(\infty)=\frac{k}{\epsilon} \simeq\left\langle 2 E_{q}\right\rangle \sim 2 M
$$

from Eqs.(5.17), (5.19) and (5.20).

We examine the dependence of the effective quark mass $M$ within the possible variation of the effective quark mass, $350 \mathrm{MeV} \lesssim M<\infty$, because even the massless quark has a large effective mass about $350 \mathrm{MeV}$ due to the dynamical chiral-symmetry breaking. From Eqs.(5.17) and (5.20), one finds that $\epsilon$ is almost inversely proportional to $M$, and the string tension $k$ does not strongly depend on $\epsilon$ and $M$ as long as $\epsilon \ll m_{B}, m_{\chi}$. One also finds that the saturated value $V_{\text {linear }}^{\text {sc }}(\infty)$ is almost proportional to the quark mass $M$ from Eq.(5.21). These relations can be examined by the use of the lattice QCD simulation for the various bare quark mass. 
The infrared screening effect on the quark potential has been observed in the lattice QCD simulation with the dynamical quarks [24]. However, most studies have been based on the small-size lattice, for instance $8^{3} \times 4$, and the finite size effect may spoil the quantitative argument on the long-range screening effect. Actually, rather different results are reported on the screening effect in the lattice QCD data [24]. It is desirable to investigate this problem by using a large-size lattice with high statistics.

\section{The Schwinger-Dyson Equation and the Dynamical Chiral-Symmetry Breaking}

In this chapter, we study the dynamical chiral-symmetry breaking by using the dual Ginzburg-Landau theory. In particular, we are interested in the relation between the color confinement and the dynamical chiral-symmetry breaking because there is a remarkable coincidence on the critical temperatures of the deconfinement phase transition and the chiral-symmetry restoration in the lattice QCD simulation with dynamical quarks [44]. To this end, we derive the Schwinger-Dyson equation for the light quarks by using the diagonal-gluon propagator $D_{\mu \nu}^{\mathrm{sc}}(k)$ in Eq.(5.12) in the dual Ginzburg-Landau theory.

Several years ago, Baker et al. [45, 46] studied the relation between the dynamical chiral-symmetry breaking and monopole condensation in the dual QCD model [47], and obtained that monopole condensation contributes to the chiral symmetry breaking. However, they used a wrong sign $[45,46]$ for the integrand in the Schwinger-Dyson equation, which was pointed out by the Kanazawa group [40]. Since this error was fatal as was shown in erratum in Ref.[45], their conclusion is no more creditable. Very recently, the Kanazawa group studied this subject by using the dual Ginzburg-Landau theory, and they encountered a difficulty in solving the Schwinger-Dyson equation in relation with the appearance of the infrared double pole in the gluon propagator [40]. They used an artificial replacement (4.18) to avoid the infrared double pole $[15,40]$, and used an angle average for the direction of the Dirac string $n_{\mu}[40,46]$,

$$
\frac{1}{2 \pi^{2}} \int d \Omega_{n} \frac{1}{(n \cdot k)^{2}}=-\frac{2}{k^{2}} .
$$

However, the inverse of the sign takes place in this replacement because the integrand in the left-hand side is positive definite. They solved the Schwinger-Dyson equation by using the above replacement, and found that the dynamical chiralsymmetry breaking is weakened by QCD-monopole condensation [40]. Their result 
seems unexpected and against the results on the relationship between the deconfinement phase transition and the chiral-symmetry restoration in the lattice QCD [44]. We speculate that the inverse of the sign in the replacement (6.1) is unnatural and problematic, and gives the origin of their unnatural result.

We shall now study this issue by using the gluon propagator including the infrared screening effect in the dual Ginzburg-Landau theory. Although the Kanazawal group has neglected the dynamical effect of light quarks, it is desirable, as an important ingredient, to include the dynamical effect of quarks such as the $q-\bar{q}$ pair creation in this subject, because the light dynamical quarks should be introduced for the study of the dynamical chiral-symmetry breaking. Hence, we use the modified diagonal-gluon propagator $D_{\mu \nu}^{\mathrm{sc}}(k)$ in Eq.(5.12), which includes the infrared screening effect. It is notable that the infrared double pole in the gluon propagator is naturally disappeared by the infrared screening effect. (See Eq.(5.12).) Hence, there is no difficulty of the infrared double pole in the gluon propagator, and we can formulate the Schwinger-Dyson equation in a straightforward way.

We study the dynamical chiral-symmetry breaking by using the SchwingerDyson equation for the quark field in the chiral limit within the rainbow approximation $[25,26]$,

$$
S_{q}^{-1}\left(p_{M}\right)=\not p_{M}+\int \frac{d^{4} k_{M}}{i(2 \pi)^{4}} \vec{Q}^{2} \gamma^{\mu} S_{q}\left(k_{M}\right) \gamma^{\nu} D_{\mu \nu}^{\mathrm{sc}}\left(k_{M}-p_{M}\right),
$$

where $S_{q}\left(p_{M}\right)$ denotes the quark propagator with the Minkowski momentum $p_{M}$. In this equation, the coupling strength between the quark and the diagonal-gluons is given by $\vec{Q}^{2}=\frac{N_{c}-1}{2 N_{c}} e^{2}$ in the $\mathrm{SU}\left(N_{c}\right)$ gauge theory [40]. We consider a simple quark propagator $S_{q}(p)$ including the momentum-dependent mass $M\left(-p_{M}^{2}\right)$,

$$
S_{q}\left(p_{M}\right)^{-1}=\not p_{M}-M\left(-p_{M}^{2}\right)+i \epsilon
$$

although the quark propagator should be taken, in a rigorous sense, as $S_{q}\left(p_{M}\right)^{-1}=A \not p_{M}+B+C \not p_{M} \not n+D \not n+i \epsilon$, where $A, B, C$, and $D$ are scalar functions of $p_{M}^{2}$ and $p_{M} \cdot n$ [46]. The Schwinger-Dyson equation for the dynamical quark mass $M\left(-p_{M}^{2}\right)$ is obtained by taking the trace of Eq.(6.2),

$$
M\left(p^{2}\right)=\int \frac{d^{4} k}{(2 \pi)^{4}} \vec{Q}^{2} \frac{M\left(k^{2}\right)}{k^{2}+M^{2}\left(k^{2}\right)} D_{\mu}^{\mu \mathrm{sc}}(k-p)
$$

after the Wick rotation in the $k_{0}$-plane and the transformation into the Euclidean 
variables, which are simply denoted as $p$ or $k$. In the Lorentz gauge, one finds

$$
\begin{aligned}
D_{\mu}^{\mu \mathrm{sc}}(k) & =\frac{1}{(n \cdot k)^{2}+\epsilon^{2}} \cdot \frac{1}{k^{2}} \cdot \frac{2 m_{B}^{2}}{k^{2}+m_{B}^{2}}\left\{k^{2}-(n \cdot k)^{2}\right\}+\frac{3+\alpha_{e}}{k^{2}} \\
& =\frac{2}{(n \cdot k)^{2}+\epsilon^{2}}\left(\frac{m_{B}^{2}}{k^{2}+m_{B}^{2}}+\frac{\epsilon^{2}}{k^{2}}-\frac{\epsilon^{2}}{k^{2}+m_{B}^{2}}\right)+\frac{2}{k^{2}+m_{B}^{2}}+\frac{1+\alpha_{e}}{k^{2}},
\end{aligned}
$$

and the Schwinger-Dyson equation becomes

$$
\begin{aligned}
M\left(p^{2}\right) & =\int \frac{d^{4} k}{(2 \pi)^{4}} \vec{Q}^{2} \frac{M\left(k^{2}\right)}{k^{2}+M^{2}\left(k^{2}\right)} \\
& \times\left(\frac{2}{\tilde{k}^{2}+m_{B}^{2}}+\frac{1+\alpha_{e}}{\tilde{k}^{2}}+\frac{2}{(n \cdot \tilde{k})^{2}+\epsilon^{2}}\left[\frac{m_{B}^{2}}{\tilde{k}^{2}+m_{B}^{2}}+\frac{\epsilon^{2}}{\tilde{k}^{2}}-\frac{\epsilon^{2}}{\tilde{k}^{2}+m_{B}^{2}}\right]\right)
\end{aligned}
$$

with $\tilde{k}_{\mu} \equiv k_{\mu}-p_{\mu}$. One finds that no infrared double pole appears in Eq.(6.6), so that one can solve it numerically without any difficulties related to the unphysical infrared divergence.

We can perform the angle integration of the first and second terms in the bracket in Eq.(6.6) by the use of the formula,

$$
\begin{aligned}
I_{1} & \equiv \int \frac{d^{4} k}{(2 \pi)^{4}} \frac{1}{\tilde{k}^{2}+c^{2}} f\left(k^{2}\right) \\
& =\frac{1}{(2 \pi)^{3}} \int_{0}^{\infty} k^{2} d k^{2} f\left(k^{2}\right) \int_{0}^{\pi} d \theta \frac{\sin ^{2} \theta}{k^{2}+p^{2}+c^{2}-2 k p \cos \theta} \\
& =\frac{1}{8 \pi^{2}} \int_{0}^{\infty} k^{2} d k^{2} f\left(k^{2}\right) \frac{1}{k^{2}+p^{2}+c^{2}+\sqrt{\left(k^{2}+p^{2}+c^{2}\right)^{2}-4 k^{2} p^{2}}}
\end{aligned}
$$

where $f\left(k^{2}\right)$ and $c$ are an arbitrary function of $k^{2}$ and an arbitrary constant, respectively. As for the remaining part in Eq.(6.6), we can partially perform the angle integration using the formula, 


$$
\begin{aligned}
I_{2} & \equiv \int \frac{d^{4} k}{(2 \pi)^{4}} \frac{1}{(n \cdot \tilde{k})^{2}+\epsilon^{2}} \cdot \frac{1}{\tilde{k}^{2}+c^{2}} f\left(k^{2}\right) \\
& =\int_{-\infty}^{\infty} \frac{d k_{n}}{2 \pi} \int_{0}^{\infty} \frac{k_{T}^{2} d k_{T}}{(2 \pi)^{2}} f\left(k^{2}\right) \frac{1}{\tilde{k}_{n}^{2}+\epsilon^{2}} \int_{-1}^{1} \frac{d(\cos \alpha)}{\tilde{k}_{n}^{2}+k_{T}^{2}+p_{T}^{2}+c^{2}-2 k_{T} p_{T} \cos \alpha} \\
& =\frac{1}{16 \pi^{3} p_{T}} \int_{-\infty}^{\infty} d k_{n} \int_{0}^{\infty} d k_{T} k_{T} f\left(k^{2}\right) \frac{1}{\tilde{k}_{n}^{2}+\epsilon^{2}} \ln \left(\frac{\tilde{k}_{n}^{2}+\left(k_{T}+p_{T}\right)^{2}+c^{2}}{\tilde{k}_{n}^{2}+\left(k_{T}-p_{T}\right)^{2}+c^{2}}\right) \\
& =\frac{1}{32 \pi^{3} p_{T}} \int_{0}^{\infty} d k^{2} f\left(k^{2}\right) \int_{-k}^{k} d k_{n} \frac{1}{\tilde{k}_{n}^{2}+\epsilon^{2}} \ln \left(\frac{\tilde{k}_{n}^{2}+\left(k_{T}+p_{T}\right)^{2}+c^{2}}{\tilde{k}_{n}^{2}+\left(k_{T}-p_{T}\right)^{2}+c^{2}}\right),
\end{aligned}
$$

where we have used the relation,

$$
\int_{-\infty}^{\infty} d k_{n} \int_{0}^{\infty} d k_{T} k_{T}=\int_{-\infty}^{\infty} d k_{n} \int_{\left|k_{n}\right|}^{\infty} d k k=\int_{0}^{\infty} d k k \int_{-k}^{k} d k_{n} .
$$

Then, after some complicated calculation on the angle integrations, one obtains a expression for the Schwinger-Dyson equation,

$$
\begin{aligned}
M\left(p^{2}\right) & =\int_{0}^{\infty} \frac{d k^{2}}{16 \pi^{2}} \frac{\vec{Q}^{2} M\left(k^{2}\right)}{k^{2}+M^{2}\left(k^{2}\right)}\left(\frac{4 k^{2}}{k^{2}+p^{2}+m_{B}^{2}+\sqrt{\left(k^{2}+p^{2}+m_{B}^{2}\right)^{2}-4 k^{2} p^{2}}}\right. \\
& +\frac{\left(1+\alpha_{e}\right) k^{2}}{\max \left(k^{2}, p^{2}\right)}+\frac{1}{\pi p_{T}} \int_{-k}^{k} d k_{n} \frac{1}{\tilde{k}_{n}^{2}+\epsilon^{2}} \\
& \left.\times\left[\left(m_{B}^{2}-\epsilon^{2}\right) \ln \left\{\frac{\tilde{k}_{n}^{2}+\left(k_{T}+p_{T}\right)^{2}+m_{B}^{2}}{\tilde{k}_{n}^{2}+\left(k_{T}-p_{T}\right)^{2}+m_{B}^{2}}\right\}+\epsilon^{2} \ln \left\{\frac{\tilde{k}_{n}^{2}+\left(k_{T}+p_{T}\right)^{2}}{\tilde{k}_{n}^{2}+\left(k_{T}-p_{T}\right)^{2}}\right\}\right]\right),
\end{aligned}
$$

where $\tilde{k}_{n} \equiv k_{n}-p_{n}, k_{T} \equiv\left(k^{2}-k_{n}^{2}\right)^{1 / 2}$. Here, the right hand side of Eq.(6.10) is a function of $p_{n}$ and $p_{T}$ generally, so that Eq.(6.10) is not a closed equation, which is brought due to the $n_{\mu}$-dependence of the diagonal gluon propagator in Eq.(5.12). To solve Eq.(6.10), we use the ansatz,

$$
p_{n}=p \sin \theta, \quad p_{T}=p \cos \theta
$$

with an angle parameter $\theta$, and then the Schwinger-Dyson equation (6.10) can be solved for each value of $\theta$. It is notable that the right hand side of Eq.(6.10) is always non-negative, which supports the existence of a nontrivial solution. 
In solving the Schwinger-Dyson equation, we use the Higashijima-Miransky approximation $[25,26]$ with a hybrid type of the running coupling constant $[40,48]$

$$
\tilde{e}=e\left(\max \left\{p^{2}, k^{2}\right\}\right), \quad e^{2}\left(p^{2}\right)=\frac{48 \pi^{2}\left(N_{c}+1\right)}{\left(11 N_{c}-2 N_{f}\right) \ln \left\{\left(p^{2}+p_{c}^{2}\right) / \Lambda_{\mathrm{QCD}}^{2}\right\}},
$$

and replace the gauge coupling constant $e$ with $\tilde{e}$ in Eq.(6.10). Here, $\Lambda_{\mathrm{QCD}}$ is the QCD scale parameter $\left(\Lambda_{\mathrm{QCD}}(\overline{\mathrm{MS}})=220 \pm 15 \pm 50 \mathrm{MeV}\right.$, Exp. $)$ [3] and $p_{c}$ is given by

$$
p_{c}^{2}=\Lambda_{\mathrm{QCD}}^{2} \exp \left[\frac{48 \pi^{2}}{e^{2}} \cdot \frac{N_{c}+1}{\left(11 N_{c}-2 N_{f}\right)}\right] .
$$

Such a treatment of the gauge coupling constant, $\tilde{e}$ in Eq.(6.12), approximately divides the momentum region into the ultraviolet part $p>p_{c}$ and the infrared part $p<p_{c}$. In the high momentum region, $p^{2} \gg p_{c}^{2}$, gauge coupling strength in Eq.(6.10) is reduced to the perturbative one in Eq.(1.1),

$$
\frac{\vec{Q}^{2}\left(p^{2}\right)}{4 \pi}=\frac{N_{c}-1}{2 N_{c}} \cdot \frac{e^{2}\left(p^{2}\right)}{4 \pi} \simeq \frac{N_{c}^{2}-1}{2 N_{c}} \frac{12 \pi}{\left(11 N_{c}-2 N_{f}\right) \ln \left(p^{2} / \Lambda_{\mathrm{QCD}}^{2}\right)}=\frac{N_{c}^{2}-1}{2 N_{c}} \alpha_{s}\left(p^{2}\right),
$$

and therefore Eq.(6.10) is reduced to a simple expression in the ultraviolet limit $p^{2} \rightarrow \infty$,

$$
M\left(p^{2}\right)=\frac{N_{c}^{2}-1}{2 N_{c}} \int_{0}^{\infty} \frac{d k^{2}}{4 \pi} \alpha_{s}\left(\max \left\{k^{2}, p^{2}\right\}\right) \frac{M\left(k^{2}\right)}{k^{2}+M^{2}\left(k^{2}\right)} \cdot \frac{\left(3+\alpha_{e}\right) k^{2}}{\max \left(k^{2}, p^{2}\right)},
$$

which coincides with the Schwinger-Dyson equation by using the free gluon field $[25,26]$. Thus, the full gluon contribution is taken into account in this ultraviolet region by the use of $\tilde{e}$ in Eq.(6.12) [40]. On the other hand, $e\left(p^{2}\right)$ in Eq.(6.12) becomes a constant in the low momentum region, $p^{2} \lesssim p_{c}^{2}$,

$$
e^{2}\left(p^{2}\right) \simeq \frac{48 \pi^{2}\left(N_{c}+1\right)}{\left(11 N_{c}-2 N_{f}\right) \ln \left(p_{c}^{2} / \Lambda_{\mathrm{QCD}}^{2}\right)}=e^{2},
$$

which seems underestimation of the strong-coupling nature in comparison with the perturbative result in Eq.(1.1). It should be noted that the dual Ginzburg-Landau theory includes QCD-monopole condensation as a nonperturbative effect related to the color confinement, which would be brought by the strong coupling in the infrared region. 
We briefly show our thinking on the nonperturbative QCD physics in terms of the dual Ginzburg-Landau theory. Owing to the asymptotic freedom of QCD, the perturbation theory is valid in the ultraviolet region, while the dynamics in the infrared region should be nonperturbative. In the dual Ginzburg-Landau theory, these two regions are approximately divided by the energy scale of the dual Meissner effect, the mass of the dual-gauge field $m_{B}$ or the abelian monopole mass $m_{\chi}$, and therefore $p_{c}$ in the above argument should correspond to $m_{B}\left(m_{\chi}\right)$, i.e. $p_{c} \sim m_{B}\left(m_{\chi}\right)$, which is valid for our parameter set, $e=5.5, \Lambda_{\mathrm{QCD}}=200 \mathrm{MeV}$ and $N_{c}=N_{f}=3, p_{c} \simeq 638 \mathrm{MeV} \sim m_{B}\left(m_{\chi}\right)$. Thus, in our framework, the ultraviolet region of $p>p_{c} \sim m_{B}\left(m_{\chi}\right)$ is regarded as the perturbative region and is characterized by the running coupling constant in the perturbation theory. On the other hand, the infrared region of $p<p_{c} \sim m_{B}\left(m_{\chi}\right)$ is essentially nonperturbative and is characterized by abelian monopole condensation or the dual Meissner effect in the dual Ginzburg-Landau theory.

We study the dynamical chiral-symmetry breaking by solving the SchwingerDyson equation (6.10) numerically in the Landau gauge $\alpha_{e}=0$, where the wave function renormalization is not needed [28] and the quark propagator (6.3) is valid for $m_{B}=0$. As for the angle parameter $\theta$, we obtain similar numerical solutions for various $\theta$ and the $\theta$-dependence seems rather small, and therefore we only show the numerical results for the averaged values of $p_{n}$ and $p_{T}, p_{n}=\left\langle p_{n}\right\rangle=\frac{1}{2} p$ and $p_{T}=\left\langle p_{T}\right\rangle=\frac{\sqrt{3}}{2} p$. We show the numerical result for the dynamical quark mass $M\left(p^{2}\right)$ in Fig. 4 for $e=5.5, \Lambda_{\mathrm{QCD}}=200 \mathrm{MeV}, m_{B}=500 \mathrm{MeV}$ and $\epsilon=80 \mathrm{MeV}$. The solid curve in the space-like region, $p^{2}\left(=-p_{M}^{2}\right)>0$, is a nontrivial solution of the Schwinger-Dyson equation (6.10), where the dynamical quark mass in the infrared region, $M(0) \simeq 348 \mathrm{MeV}$, seems a reasonable value in terms of the constituent quark model [1].

We also investigate the light-quark confinement, although this issue is rather difficult because the discussion of the static quark potential cannot be applied to the light quarks unlike the heavy quarks because the light quark cannot be fixed at a spatial point. Instead, the light-quark confinement is characterized by the disappearance of the physical poles in the quark propagator, which can be obtained by the analytic continuation of the dynamical quark mass $M\left(p^{2}\right)$ to the time-like region in principle [49]. In Fig.4, the solid curve in the time-like region, $p^{2}\left(=-p_{M}^{2}\right)<0$, is obtained by a smooth extrapolation of $M\left(p^{2}\right)$ from the region $0<p^{2}<p_{c}^{2}$, assuming the continuous property of the quark propagator. We find the solid curve does not cross the line, $p^{2}\left(=-p_{M}^{2}\right)=-M^{2}\left(p^{2}\right)$, corresponding to the on-shell condition. Hence, the quark propagator has no physical poles, and the light quarks never appear as the asymptotic fields, which means the color confinement for light quarks. 
We now investigate the effect of QCD-monopole condensation on the dynamical chiral-symmetry breaking. We show in Fig.5 the numerical results of the dynamical quark mass $M\left(p^{2}\right)$ for the various values of $m_{B}$, the mass of the dual-gauge field $\vec{B}_{\mu}$. The other parameters are set to the same values as in Fig.4. No nontrivial solution is found for the small values of $m_{B}$, e.g. $m_{B} \lesssim 200 \mathrm{MeV}$. The nontrivial solution is found for $m_{B} \gtrsim 300 \mathrm{MeV}$, and the dynamical quark mass $M\left(p^{2}\right)$ becomes larger at each $p^{2}$ as $m_{B}$ gets larger, and therefore QCD-monopole condensation provides a large contribution to the dynamical chiral-symmetry breaking. Thus, we find the close relation between the color confinement and the dynamical chiralsymmetry breaking through QCD-monopole condensation in the dual GinzburgLandau theory.

We also calculate the quark condensate $\langle\bar{q} q\rangle$, and the pion decay constant $f_{\pi}$, which also characterize the dynamical chiral-symmetry breaking as well as the dynamical quark mass in the infrared limit, $M(0)$. The quark condensate is given by

$$
\langle\bar{q} q\rangle_{\Lambda}=\int^{\Lambda} \frac{d^{4} k_{M}}{i(2 \pi)^{4}} \operatorname{tr} S_{q}\left(k_{M}\right)=-\frac{N_{c}}{4 \pi^{2}} \int_{0}^{\Lambda^{2}} d k^{2} \frac{k^{2} M\left(k^{2}\right)}{k^{2}+M^{2}\left(k^{2}\right)},
$$

where ultraviolet cutoff $\Lambda$ is introduced to regularize the ultraviolet divergence of the integral. By using the asymptotic form of the quark mass $M\left(p^{2}\right)$ [50], one gets the quark condensate in the renormalization-group invariant form in the asymptotic region $[40,51]$,

$$
\langle\bar{q} q\rangle_{\mathrm{RGI}}=\frac{\langle\bar{q} q\rangle_{\Lambda}}{\left\{\ln \left(\Lambda^{2} / \Lambda_{\mathrm{QCD}}^{2}\right)\right\}^{a}}
$$

where $a=\frac{9\left(N_{c}^{2}-1\right)}{2 N_{c}\left(11 N_{c}-2 N_{f}\right)}=\frac{4}{9}$ for $N_{c}=N_{f}=3$. We calculate $\langle\bar{q} q\rangle_{\mathrm{RGI}}$ in the asymptotic region $\Lambda^{2} / \Lambda_{\mathrm{QCD}}^{2}=10^{6}$, where the electro-weak unification takes place. The pion decay constant $\left(f_{\pi}=93 \mathrm{MeV}\right.$, Exp. $)$ is given by the Pagels-Stokar formula, $[52]$

$$
f_{\pi}^{2}=\frac{N_{c}}{4 \pi^{2}} \int_{0}^{\infty} d k^{2} \frac{k^{2} M\left(k^{2}\right)}{\left\{k^{2}+M^{2}\left(k^{2}\right)\right\}^{2}}\left(M\left(k^{2}\right)-\frac{k^{2}}{2} \cdot \frac{d M\left(k^{2}\right)}{d k^{2}}\right) .
$$

The numerical results on $M(0),\langle\bar{q} q\rangle_{\mathrm{RGI}}$ and $f_{\pi}$ are shown in Fig.6 for the various values of the gauge coupling $e$ and the mass of the dual-gauge field $m_{B}$. As for the $m_{B}$ dependence on these quantities, the dynamical-symmetry breaking appears strongly for the large value of $m_{B}$, and therefore a large amount of the chiral-symmetry breaking seems to be brought by QCD-monopole condensation, 
which is responsible to the color confinement. These quantities are well reproduced as $M(0) \simeq 348 \mathrm{MeV},\langle\bar{q} q\rangle_{\mathrm{RGI}} \simeq-(192 \mathrm{MeV})^{3},\langle\bar{q} q\rangle_{\Lambda=1 \mathrm{GeV}} \simeq-(229 \mathrm{MeV})^{3}$ and $f_{\pi} \simeq$ $83.6 \mathrm{MeV}$ by using the parameter set, $e=5.5, \Lambda_{\mathrm{QCD}}=200 \mathrm{MeV}[3], m_{B}=500 \mathrm{MeV}$ and $\epsilon=80 \mathrm{MeV}$. This parameter set is consistent with the perturbative QCD in the ultraviolet region [1] and the quark potential as shown in chapter 4 and 5 . In particular, this parameter set provides the reasonable values of the running gauge coupling constant, $\alpha_{s}\left(m_{Z}^{2}\right) \simeq 0.123($ Exp., $0.108 \pm 0.005)[2]$ and $\alpha_{s}\left((35 \mathrm{GeV})^{2}\right) \simeq$ 0.146 (Exp., $0.10 \sim 0.17$ ) [1] for $N_{f}=4$. Thus, the dual Ginzburg-Landau theory provides the consistent picture for the phenomenological aspects of QCD, that is the perturbative QCD, the color confinement and the dynamical chiral-symmetry breaking.

It is notable that large values for $e$ and $\Lambda_{\mathrm{QCD}}$ are needed in the absence of QCD-monopole condensation, $m_{B}=0$, to reproduce the enough amount of the chiral-symmetry breaking, as is shown in Fig.6. This case, $m_{B}=0$, corresponds to the Schwinger-Dyson equation in Refs.[27] and [28], where the authors used large values for $e \sim 10$ and $\Lambda_{\mathrm{QCD}}=0.5 \sim 1 \mathrm{GeV}$ to reproduce $\langle\bar{q} q\rangle_{\mathrm{RGI}}$ or $f_{\pi}$. This seems inconsistent with the QCD scale parameter $\Lambda_{\mathrm{QCD}} \sim 200 \mathrm{MeV}$ obtained from the high-energy experimental data by using the perturbative QCD [3], and the gauge coupling $e$ estimated from the static quark potential. (See chapter 4.) The reason of the disagreement for $m_{B}=0$ seems rather trivial, because there is no nonperturbative effect like the color confinement in the infrared region, where the coupling constant $e$ becomes extremely strong and the nonperturbative effect should appear. Such a missing contribution in the infrared region inevitably leads the underestimation for the physical quantities like $\langle\bar{q} q\rangle$ or $f_{\pi}$. Hence, if one wants to reproduce enough values for them for $m_{B}=0$, one needs a large coupling $e \sim 10$ and a large QCD scale parameter $\Lambda_{\mathrm{QCD}}=0.5 \sim 1 \mathrm{GeV}$, which enlarges the strong-coupling region $\left(p<\Lambda_{\mathrm{QCD}}\right)$.

Finally, we consider the physical meaning of the above results on the close relation between the confinement and the dynamical chiral-symmetry breaking through QCD-monopole condensation. The dynamical chiral-symmetry breaking is characterized by the quark and antiquark pair condensation, $\langle\bar{q} q\rangle \neq 0$, which is brought by the strong attractive force between the quark and the antiquark with the opposite color charge $[7,8]$. In the dual Ginzburg-Landau theory, QCD-monopole condensation leads the linear confining potential, which bring the strong and longrange attractive force between the quark and the antiquark with the opposite color charge, and therefore quark pair condensation is expected to be realized. Thus, the dynamical chiral-symmetry breaking should be enhanced by the presence of the confining force or QCD-monopole condensation. On the other hand, only a Coulomb-type force remains between quarks when the QCD-monopole field is not condensed, $m_{B}=0$, and therefore the weak attractive force is only expected 
between the quark and the antiquark. Thus, the realization of the dynamical chiralsymmetry breaking is rather hard in the absence of QCD-monopole condensation, unless quite large values for $e$ and $\Lambda_{\mathrm{QCD}}$ are used, as shown in Fig.6.

\section{Summary and Discussions}

We have studied nonperturbative features in QCD such as the color confinement, the $q-\bar{q}$ pair creation and the dynamical chiral-symmetry breaking in terms of QCD-monopole condensation by using the dual Ginzburg-Landau theory. We have investigated the appearance of QCD-monopoles in QCD in the abelian gauge proposed by 't Hooft. The nonabelian nature leads to the nontrivial homotopy class, $\pi_{2}\left(\mathrm{SU}\left(N_{c}\right) /[\mathrm{U}(1)]^{N_{c}-1}\right)=\pi_{1}\left([\mathrm{U}(1)]^{N_{c}-1}\right)=Z_{\infty}^{N_{c}-1}$, which provides the origin of the QCD-monopoles. As a phenomenological theory of the nonperturbative QCD, we have constructed the dual Ginzburg-Landau theory including the confining mechanism in terms of the dual Meissner effect by QCD-monopole condensation. As for the heavy quark confinement, we have derived the static quark potential including the linear part and the Yukawa part in the dual Ginzburg-Landau theory within the quenched approximation. We have derived a simple formula for the string tension, which is analogous to the energy per unit length of the vortex in the superconductivity.

We have studied the dynamical effect of light quarks on the quark potential with respect to the infrared screening effect brought by the $q-\bar{q}$ pair creation or the cut of the hadronic strings. We have estimated the screening length, $R_{s c} \simeq 1 \mathrm{fm}$, for the quark confining potential by the use of the Schwinger formula for the $q-\bar{q}$ pair creation. The corresponding infrared cutoff has been introduced to the strong longrange correlation part in the gluon propagator, and we have obtained a compact formula for the quark potential including the screening effect in the infrared region.

We have investigated the dynamical chiral-symmetry breaking in the dual Ginzburg-Landau theory by the use of the Schwinger-Dyson equation with the gluon propagator including the dual Meissner effects or the nonperturbative effect related to the color confinement. In our approach, the nonperturbative region and the perturbative region are naturally divided by the energy scale of QCD-monopole condensation, and characterized by the dual Meissner effect and the perturbative running coupling constant, respectively. We have found a large enhancement of the dynamical chiral-symmetry breaking due to QCD-monopole condensation, which supports the close relation between the color confinement and the chiral-symmetry breaking through QCD-monopole condensation.

The dynamical quark mass, the pion decay constant and the quark condensate are well reproduced by using the consistent values of the gauge coupling constant 
and the QCD scale parameter with the perturbative QCD and the quark confining potential. By using the parameter set, $e=5.5, \Lambda_{\mathrm{QCD}}=200 \mathrm{MeV}, m_{B}=500 \mathrm{MeV}$ and $\epsilon=80 \mathrm{MeV}$, we have obtained good results on both the quark confining potential and the dynamical chiral-symmetry breaking, e.g. $M(0) \simeq 348 \mathrm{MeV},\langle\bar{q} q\rangle_{\mathrm{RGI}} \simeq$ $-(192 \mathrm{MeV})^{3},\langle\bar{q} q\rangle_{\Lambda=1 \mathrm{GeV}} \simeq-(229 \mathrm{MeV})^{3}$, and $f_{\pi} \simeq 83.6 \mathrm{MeV}$. This parameter set also provides the reasonable values of the running gauge coupling constant, $\alpha_{s}\left(m_{Z}^{2}\right) \simeq 0.123($ Exp. $0.108 \pm 0.005)$ and $\alpha_{s}\left((35 \mathrm{GeV})^{2}\right) \simeq 0.146($ Exp., $0.10 \sim 0.17)$ for $N_{f}=4$, and is found to be consistent with the perturbative QCD in the ultraviolet region.

The color confinement of the light quarks has been also examined in the dual Ginzburg-Landau theory by the smooth extrapolation of the quark mass function $M\left(p^{2}\right)$ from the space-like region to the time-like region, and the disappearance of the physical poles in the light-quark propagator has been found.

Thus, we have obtained the consistent picture for the various phenomena in QCD by using the dual Ginzburg-Landau theory. We have shown in this theory the quark confining potential within the quenched approximation, the infrared screening effect for the quark potential by the light quark-pair creation, the dynamical chiral-symmetry breaking by using the consistent value of $\Lambda_{\mathrm{QCD}}$ with the perturbative QCD, and the light-quark confinement.

We now discuss the other interesting subjects in QCD in terms of QCDmonopole condensation in the dual Ginzburg-Landau theory. It is meaningful to examine the relation between abelian monopole condensation and the QCD phase transition, which is characterized by the confinement and the chiral symmetry. Due to the asymptotic freedom of QCD, the gauge coupling constant becomes small at high temperatures, and one expects the phase transition from the nonperturbative vacuum to the perturbative vacuum, which corresponds to the deconfinement phase transition and the chiral-symmetry restoration [7]. The critical temperatures of these phase transitions coincide in the study of the lattice QCD simulation with light dynamical quarks [44], and the critical gauge couplings also coincide between the deconfinement and the chiral-symmetry restoration in the lattice QCD. Hence, there must be close relation between the confinement and the chiral-symmetry breaking.

In the dual Ginzburg-Landau theory, the QCD phase transition is characterized by the presence or the absence of QCD-monopole condensation. In the strongcoupling case or the low-temperature case, we expect QCD-monopole condensation, which results in the color confinement and the dynamical chiral-symmetry breaking. At a critical coupling or a critical temperature, such QCD-monopole condensation would disappear, and therefore one expects not only the disappearance of the color confinement, e.g. the dual Meissner effect or the confining force between 
quarks, but also the large reduction of the dynamical chiral-symmetry breaking. In particular, the dynamical chiral-symmetry breaking also vanishes for $e \lesssim 7$ when the QCD-monopole condensate disappears, $m_{B}=0$. (see Fig.6.) Thus, we expect the coincidence of the deconfinement phase transition and the chiral-symmetry restoration in relation with the vanishing of QCD-monopole condensation.

We will briefly show the future program of our study in the dual GinzburgLandau theory. It is straightforward to extend the Schwinger-Dyson equation for the massive current quark, $m \neq 0$, and therefore we can also investigate the properties related to the massive quarks such as s, c and t-quark, e.g. s-quark mass $M_{s}\left(p^{2}\right), f_{K}$ and $\langle\bar{s} s\rangle$, as well as u and d-quark [27] in the dual Ginzburg-Landau theory.

The hadron properties can be studied by solving the Bethe-Salpeter equation [28] or the Faddeev equation [53] in the dual Ginzburg-Landau theory. The mass and the decay constant of mesons $\left(\sigma, \rho, A_{1}\right)$ would be calculated by using the similar technique of the ladder Bethe-Salpeter equation in Ref.[28] with the diagonalgluon propagator $D_{\mu \nu}^{\mathrm{sc}}(k)$ in Eq.(5.12), although the equation may be complicated due to its $n_{\mu}$ dependence. It would be interesting to investigate the properties of mesons including the heavy quark by using the Bethe-Salpeter equation in this scheme, because dynamics of the c-quark is hardy controlled in the lattice gauge simulation. Although the baryon property can be obtained by the Faddeev equation in principle, it would be rather complicated to solve numerically. However, approximate properties of the baryon would be calculable by regarding the baryon as the bound state of the quark and the diquark $[54,55]$.

We consider the other possible applications of the dual Ginzburg-Landau theory to the hadron physics. The nonrelativistic quark models [56] may be reformulated by using the diagonal-gluon propagator in the dual Ginzburg-Landau theory, instead of the introduction of the confining potential, which would be automatically reproduced. The chiral soliton picture [33] of the nucleon or the $\Delta(1232)$-resonance based on the nontrivial homotopy group $\pi_{2}\left(\mathrm{SU}\left(N_{f}\right) / \mathrm{U}(1)\right)=\pi_{1}(\mathrm{U}(1))=Z_{\infty}$ is one of the most interesting issue in the hadron physics, and such a chiral soliton would be investigated from the underlying quark degrees of freedom by using the $n_{\mu}$-averaged quark-current correlation in Eq.(4.2) and the similar argument to the soliton in the Nambu-Jona-Lasinio model [57].

We have divided into the nonperturbative and the perturbative regions in terms of QCD-monopole condensation in the dual Ginzburg-Landau theory. We compare such a division with the operator product expansion [58], where one introduces normalization point $\mu$, and fluctuations with virtual low momentum $p$ such as $p^{2}<$ $\mu^{2}$ are included in the local operators, while those at large virtual momentum $p^{2}>$ $\mu^{2}$ are included in the Wilson coefficients by definition [7]. Since the small scale 
physics is approximately described by the perturbation theory and nonperturbative phenomena are connected with the long scale physics, the normalization point $\mu$ physically corresponds to the border of the nonperturbative and the perturbative regions. In our framework, such a division is given by the typical energy scale of QCD-monopole condensation, $p_{c} \sim m_{B}\left(m_{\chi}\right) \sim 1 \mathrm{GeV}$, and the running coupling constant based on a perturbative scheme is used for the small scale physics, $p^{2}>p_{c}^{2}$, while the nonperturbative nature appears as QCD-monopole condensation mainly in the long scale physics, $p^{2}<p_{c}^{2}$. Thus, our framework seems similar to the operator product expansion, and then QCD-monopole condensation may provide the physical image of the separation between the two different scale regions in the operator product expansion.

Furthermore, the dual Ginzburg-Landau theory would also provide the calculation scheme for the matrix elements of the local operators, e.g. $\left\langle G_{\mu \nu} G^{\mu \nu}\right\rangle$ or $\langle\bar{q} q\rangle$, in the operator product expansion, because we have obtained the quark propagator and the diagonal-gluon propagator including the nonperturbative effect. Hence, a powerful technique should be obtained by using such a calculation for the matrix elements of the local operators and the operator product expansion in the QCD sum rule [59] in estimating the various physical quantities. Such a calculation scheme would be also applied to the estimation of the structure functions [1] of the nucleon observed in deep inelastic lepton-nucleon scattering as a useful technique for the studies of the proton spin problem [60] and the breaking of the Gottfried sum rule [61].

\section{ACKNOWLEDGEMENTS}

We are grateful to $\mathrm{H}$. Monden for her contributions on QCD-monopole condensation in the early stage of this study. We have used the program D7NLFRHM of TLIB in Tokyo Computational Center made by K.-I. Kondo, H. Nakatani and H. Mino in solving the Schwinger-Dyson equation. One of the authors (H.S.) is supported by the Special Researchers' Basic Science Program at RIKEN. 


\section{REFERENCES}

1. For instance, O. Nachtmann, "Elementary Particle Physics", (SpringerVerlag, Berlin, 1990) 1.

2. U. Amaldi, W. de Boer and H. Furstenau, Phys. Lett. B260 (1991) 447.

3. BCDMS Collaboration, A. C. Benvenuti et al., Phys. Lett. B223 (1989) 490.

4. For instance, T. P. Cheng and L. F. Li, "Gauge theory of elementary particle physics", (Clarendon press, Oxford, 1984) 1.

5. For a review article, M. Bando, T. Kugo and K. Yamawaki, Phys. Rep. 164 (1988) 217.

6. J. Gasser and H. Leutwyler, Phys. Rep. 87 (1982) 77.

7. For a review article, E. V. Shuryak, "The QCD vacuum, hadrons and the superdense matter", (World Scientific, Singapore, 1988) 1.

8. Y. Nambu and G. Jona-Lasinio, Phys. Rev. 122 (1961) 345.

9. For a recent review article, K. Sailer, Th. Schönfeld, Zs. Schram, A. Schäfer and W. Greiner, J. Phys. G: Nucl. Part. Phys. 17 (1991) 1005.

10. Y. Nambu, Phys. Rev. D10 (1974) 4262.

11. G. 't Hooft, "High Energy Physics", ed. A. Zichichi (Editorice Compositori, Bologna, 1975).

12. S. Mandelstam, Phys. Rep. C23 (1976) 245.

13. G. 't Hooft, Nucl. Phys. B190 (1981) 455.

14. T. Suzuki, Prog. Theor. Phys. 80 (1988) 929 ; 81 (1989) 752.

15. S. Maedan and T. Suzuki, Prog. Theor. Phys. 81 (1989) 229.

16. S. Maedan, Y. Matsubara and T. Suzuki, Prog. Theor. Phys. 84 (1990) 130.

S. Kamizawa, Y. Matsubara, H. Shiba and T. Suzuki, Nucl. Phys. B389 (1993) 563.

17. T. Suzuki and I. Yotsuyanagi, Phys. Rev. D42 (1990) 4257.

T. Suzuki and I. Yotsuyanagi, Nucl. Phys. B (Proc. Suppl.) 20 (1991) 236.

18. S. Hioki, S. Kitahara, S. Kiura, Y. Matsubara, O. Miyamura, S. Ohno and T. Suzuki, Phys. Lett. B272 (1991) 326.

S. Hioki, S. Kitahara, S. Ohno, T. Suzuki, Y. Matsubara and O. Miyamura, Phys. Lett. B285 (1992) 343.

19. E. Daggoto and J. Kogut, Nucl. Phys. B295 (1988) 123 and references therein. 
20. T. Banks, R. Myerson and J. Kogut, Nucl. Phys. B129 (1977) 493.

J. L. Cardy, Nucl. Phys. B170 (1980) 369.

21. T. A. DeGrand and D. Toussaint, Phys. Rev. D22 (1980) 2478.

22. A. S. Kronfeld et al., Phys. Lett. B198 (1987) 516.

A. S. Kronfeld, G. Schierholz and U. -J. Weise, Nucl. Phys. B293 (1987) 461.

23. H. Shiba and T. Suzuki, Kanazawa Univ., Report No.Kanazawa 93-9, (1993); Report No.Kanazawa 93-10, (1993).

24. For recent articles, L. I. Unger, Phys. Rev. D48 (1993) 3319.

W. Sakuler, W. Burger, M. Faber, H. Marukum, M. Muller, P. De Forcrand, A. Nakamura and I. O. Stamatescu, Phys. Lett. B276 (1992) 155.

25. K. Higashijima, Phys. Rev. D29 (1984) 1228; Prog. Theor. Phys. Suppl. 104 (1991) 1.

26. V. A. Miransky, Sov. J . Nucl. Phys. 38(2) (1983) 280.

27. A. Barducci, R. Casalbuoni, S. De Curtis, D. Dominici and R. Gatto, Phys. Lett. B193 (1987) 305.

A. Barducci, R. Casalbuoni, S. De Curtis, D. Dominici and R. Gatto, Phys. Rev. D38 (1988) 238.

28. T. Kugo and M. G. Mitchard, Phys. Lett. B286 (1992) 335.

K-I. Aoki, T. Kugo and M. G. Mitchard, Phys. Lett. B266 (1991) 467.

K-I. Aoki, M. Bando, T. Kugo, M. G. Mitchard and H. Nakatani, Prog. Theor. Phys. 84 (1990) 683.

29. H. Suganuma and T. Tatsumi, Phys. Lett. B269 (1991) 371.

H. Suganuma and T. Tatsumi, Prog. Theor. Phys. 90 (1993) 379.

30. H. Suganuma and T. Tatsumi, Ann. Phys. (N.Y.) 208 (1991) 470.

31. G. 't Hooft, Nucl. Phys. B79 (1974) 276.

A. M. Polyakov, JETP Lett. 20 (1974) 194.

32. For instance, R. Rajaraman, "Solitons and Instantons", (North-Holland, Amsterdam, 1982) 1 and references therein.

33. V.G.Makhoankov, Y. P. Rybakov and V. I. Sanyuk, "The Skyrme model", (Springer-Verlag, Berlin Heidelberg, 1993.) 1 and references therein.

34. D. Zwanziger, Phys. Rev. D3 (1971) 880.

35. M. Blagojevic and P. Senjanovic, Nucl. Phys. B161 (1979) 112.

36. K. Bardacki and S. Samuel, Phys. Rev. D18 (1978) 2849.

S. Samuel, Nucl. Phys. B154 (1979) 62. 
37. E. M. Lifshitz and L. P. Pitaevsii, Vol.9 of Course of Theoretical Physics, "Statistical Physics, Part 2", (Pergamon press, Oxford, 1981) 1.

38. H. B. Nielsen and P. Olesen, Nucl. Phys.B61 (1973) 45.

39. K. Huang, "Quarks, Leptons and Gauge Fields", World Scientific, Singapore (1982) 1.

40. S. Kamizawa, Doctor thesis, Kanazawa Univ., (1993).

41. For a recent review article, W. Lucha, F. F. Schöberl and D. Gromes, Phys. Rep. 200, No.4 (1991) 127.

42. For instance, C. Itzykson and J. B. Zuber, "Quantum Field Theory", (McGraw-Hill, New York, 1985) 1.

43. N. K. Glendenning and T. Matsui, Phys. Rev. D28 (1983) 2890; Phys. Lett. B141 (1984) 419.

44. For instance, B. Petersson, Nucl. Phys. A525 (1991) 273c; published in Amsterdam Lattice 1992 (1992) 66 and references therein.

45. M. Baker, J. S. Ball and F. Zachariasen, Phys. Rev. D38 (1988) 1926; Erratum-ibid. D47 (1993) 743.

46. G. Krein and A. G. Williams, Phys. Rev. D43 (1991) 3541.

47. For a recent review article, M. Baker, J. S. Ball and F. Zachariasen, Phys. Rep. 209 (1991) 73.

48. D. Atkinson and P. W. Johnson, Phys. Rev. D37 (1988) 2296.

49. For the QED version, R. Fukuda and T. Kugo, Nucl. Phys. B117 (1976) 250.

50. K. Lane, Phys. Rev. D10 (1974) 2605.

H. D. Politzer, Nucl. Phys. B117 (1976) 397.

H. Pagels, Phys. Rev. D19 (1979) 3080.

51. G. Krein, P. Tang and A. G. Williams, Phys. Lett. B215 (1988) 145.

52. H. Pagels and S. Stoker, Phys. Rev. D20 (1979) 2947; D22 (1980) 2876.

53. For a recent review article, V. B. Belyaev, "Lectures on the Theory of Few-Body Systems", (Springer-Verlag, Berlin ,1990) 1.

54. Papers in Proc. of the Workshop on "Diquarks", ed. M. Anselmino et al. (World Scientific, Singapore, 1988) 1.

55. B. Stech, Phys. Rev. D36 (1987) 975; Nucl. Phys. B(Proc. Suppl.)7a (1989) 106.

56. For a recent review article, S. N. Mukherjee, R. Nag, S. Sanyal, T. Morii, J. Morishita and M. Tsuge, Phys. Rep. 231 (1993) 201. 
57. T. Meissner, F. Grummer and K. Goeke, Phys. Lett. B227 (1989) 296.

58. K. G. Wilson, Phys. Rev. 179 (1969) 1499.

59. M. A. Shifman, A. I. Vainshtein and V. I. Zakharov, Nucl. Phys. B147 (1979) 385, 448 and 519.

L. J. Reinders, H. Rubinstein and S. Yazaki, Phys. Rep. 127 (1985) 1 and references therein.

60. R. L. Jaffe and A. Manohar, Nucl. Phys. B337 (1990) 509.

61. New Muon Collaboration, Phys. Rev. Lett. 66 (1991) 2717 and references therein.

\section{FIGURE CAPTIONS}

1) The static quark potential $V(r)$ in the dual Ginzburg-Landau theory in the quenched approximation. The horizontal axis denotes the distance between a static quark-antiquark pair. The solid curve is the result for $\mathrm{e}=5.5, m_{B}=500$ $\mathrm{MeV}$ and $m_{\chi}=1.26 \mathrm{GeV}$. The dashed curve denotes the Cornell potential with $e_{C}=2.0$ and $k_{C}=1.0 \mathrm{GeV} / \mathrm{fm}$.

2) The normalized pair creation rate $w\left(p_{T}\right) / w_{s c}$ as a function of the energy of the created $q-\bar{q}$ pair for $M=350 \mathrm{MeV}$ and $k=1.0 \mathrm{GeV} / \mathrm{fm}$. The horizontal axis denotes the quark pair energy, $2 E_{q}\left(p_{T}\right)=2\left(p_{T}^{2}+M^{2}\right)^{1 / 2}$. The expectation value of the quark pair energy is found to be $\left\langle 2 E_{q}\left(p_{T}\right)\right\rangle \simeq 850 \mathrm{MeV}$.

3) The infrared screening effect on the quark confining potential $V_{\text {linear }}^{s c}(r)$ for several values of the infrared cutoff $\epsilon$. The QCD-monopole mass $m_{\chi}$ is chosen to reproduce the string tension, $k=1.0 \mathrm{GeV} / \mathrm{fm}: m_{\chi}=1.26,1.15$ and $1.01 \mathrm{GeV}$ for $\epsilon=0,100$ and $200 \mathrm{MeV}$, respectively. One finds the saturation behavior of the confining potential in the long distance.

4) The squared value of the dynamical quark mass, $M\left(p^{2}\right)$, as a function of the Euclidean momentum squared $p^{2}=-p_{M}^{2}$ for $e=5.5, \Lambda_{\mathrm{QCD}}=200 \mathrm{MeV}$, $m_{B}=500 \mathrm{MeV}$ and $\epsilon=80 \mathrm{MeV}$. The solid curve in the space-like region, $p^{2}>$ 0 , denotes the numerical solution of the Schwinger-Dyson equation. The solid curve in the time-like region, $p^{2}<0$, is obtained by its smooth extrapolation. The dotted straight line denotes the on-shell state $M^{2}\left(p^{2}\right)=-p^{2}$. One finds the solid curve does not cross this dotted straight line, which means the disappearance of the pole in the quark propagator.

5) The dynamical quark mass $M\left(p^{2}\right)$ as a function of the Euclidean momentum squared $p^{2}=-p_{M}^{2}$ for several values of the mass of the dual-gauge field $\vec{B}_{\mu}$, $m_{B}=300,400$ and $500 \mathrm{MeV}$. The other parameters are set to the same values as in Fig.4. No nontrivial solution is found for the small values of $m_{B}$, e.g. 
$m_{B} \lesssim 200 \mathrm{MeV}$. The quark mass $M\left(p^{2}\right)$ takes larger value at each $p^{2}$ as $m_{B}$ gets larger, which physically means that QCD-monopole condensation contributes to the dynamical chiral-symmetry breaking.

6) The physical quantities related to the dynamical chiral-symmetry breaking as a function of the gauge coupling constant of $\mathrm{QCD}, e$, for several values of the dual-gauge field $m_{B}$ : (a) the dynamical quark mass at $p=0, M(0)$, (b) the quark condensate $\langle\bar{q} q\rangle_{\mathrm{RGI}}$, and (c) the pion decay constant $f_{\pi}$. One finds that these quantities are well reproduced for the parameter set $(e=$ $5.5, \Lambda_{\mathrm{QCD}}=200 \mathrm{MeV}, m_{B}=500 \mathrm{MeV}, \epsilon=80 \mathrm{MeV}$ ), which is consistent with the confining property and the perturbative QCD. On the contrary, large values for $e$ and $\Lambda_{\mathrm{QCD}}$ are needed to reproduce the magnitude of the chiralsymmetry breaking for $m_{B}=0$. 
This figure "fig1-1.png" is available in "png" format from: http://arxiv.org/ps/hep-ph/9312350v2 
This figure "fig2-1.png" is available in "png" format from: http://arxiv.org/ps/hep-ph/9312350v2 
This figure "fig3-1.png" is available in "png" format from: http://arxiv.org/ps/hep-ph/9312350v2 
This figure "fig1-2.png" is available in "png" format from: http://arxiv.org/ps/hep-ph/9312350v2 
This figure "fig2-2.png" is available in "png" format from: http://arxiv.org/ps/hep-ph/9312350v2 
This figure "fig3-2.png" is available in "png" format from: http://arxiv.org/ps/hep-ph/9312350v2 
This figure "fig1-3.png" is available in "png" format from: http://arxiv.org/ps/hep-ph/9312350v2 
This figure "fig2-3.png" is available in "png" format from: http://arxiv.org/ps/hep-ph/9312350v2 